\title{
A collision model for grain-resolving simulations of flows over dense, mobile, polydisperse granular sediment beds
}

\author{
Edward Biegert ${ }^{1, a}$, Bernhard Vowinckel ${ }^{2, a}$, Eckart Meiburg $^{3, a}$ \\ ${ }^{a}$ Department of Mechanical Engineering, University of California, Santa Barbara, CA, USA
}

\begin{abstract}
We present a collision model for phase-resolved Direct Numerical Simulations of sediment transport that couple the fluid and particles by the Immersed Boundary Method. Typically, a contact model for these types of simulations comprises a lubrication force for particles in close proximity to another solid object, a normal contact force to prevent particles from overlapping, and a tangential contact force to account for friction. Our model extends the work of previous authors to improve upon the time integration scheme to obtain consistent results for particle-wall collisions. Furthermore, we account for polydisperse spherical particles and introduce new criteria to account for enduring contact, which occurs in many sediment transport situations. This is done without using arbitrary values for physically-defined parameters and by maintaining the full momentum balance of a particle in enduring contact. We validate our model against several test cases for binary particle-wall collisions as well as the collective motion of a sediment bed sheared by a viscous flow, yielding satisfactory agreement with experimental data by various authors.
\end{abstract}

Keywords: Direct Numerical Simulations, Immersed Boundary Method, contact modeling, particle-laden flow, sediment transport

\footnotetext{
${ }^{1}$ ebiegert@engineering.ucsb.edu

2 vowinckel@engineering.ucsb.edu

${ }^{3}$ meiburg@engineering.ucsb.edu
} 


\section{Introduction}

The flow over dense, mobile granular beds plays a central role in multiple applications in environmental, mechanical, and process engineering. Prime examples of this type of problem are turbidity currents and powder snow avalanches 5 (Meiburg and Kneller, 2010), for which resuspension of particles essentially determines the dynamics of the flow. The resuspension threshold is quantified by the ratio of hydrodynamic drag and lift forces to the weight of the particles, known as the Shields number (Shields, 1936). The critical Shields parameter, however, has proven to be a poor predictor for the onset of particle erosion (Garcia, 2008), and substantial efforts have been made in hydraulic engineering to overcome this difficulty (Seminara, 2010). To date, progress has been slow due to the experimental difficulty of measuring dense particle-laden flows in a laboratory. In recent years, numerical simulations have provided an alternative way to study fluid-particle interactions under these complex conditions. In particular, the Direct Numerical Simulation (DNS) of particle-laden flows using the Immersed Boundary Method (IBM) has become a very attractive option. A main advantage of this approach is that it allows for a fully-coupled system by accounting for particle-fluid, fluid-particle, and particle-particle interactions. While coupling between the particle and the fluid can be realized by a straightforward implementation of an IBM, particle-particle coupling has to be modeled by suitable expressions for the collision processes involved.

A number of different phase-resolving methods to simulate mobile particles in a viscous flow have been developed in the past two decades. Glowinski et al. (1999) and Patankar et al. 2000 developed a Distributed Lagrange Multiplier (DLM)/fictitious domain approach that forces the fluid throughout the volume of the particle to move in a rigid body motion with the particle. While this method was designed for a finite element framework, Kajishima et al. (2001) and Apte et al. 2009) later developed different versions to be used in a finite difference framework. More recently, other methods that enforce the no-slip condition on the particle surface have been developed. Zhang and Prosperetti 
2005 developed PHYSALIS, which uses the analytical solution for Stokes flow around a sphere. Uhlmann (2005) developed an IBM that enforces the no-slip condition using interpolation and spreading operations via Dirac delta functions. Kempe and Fröhlich (2012b extended this method to make it stable for a larger

35 range of particle/fluid density ratios. Although there exists a variety of phaseresolving methods, the choice of collision models, on the other hand, has not been as diverse. Derksen 2011) and Derksen (2015) used a hard-sphere model, which resolves collisions instantaneously. Glowinski et al. (1999) have developed a repulsive potential $(\mathrm{RP})$ model that prevents particles from overlapping by

40 applying a repulsive force at some small distance before the particles come in contact. Many other authors have adopted this model for simulations involving dilute suspensions of particles. For example, Uhlmann (2008) and Santarelli and Fröhlich (2015) investigated particles in a vertical turbulent channel flow, Lucci et al. (2010) studied the impact of finite size particles on isotropic turbu-

45 lence, and Breugem (2012) and Picano et al. (2015) have presented results for a horizontal flow laden with neutrally-buoyant particles. In these simulations, particles rarely came in contact, and thus were successfully governed mostly by the IBM, using the repulsive potential only to prevent overlap.

For the situation involving shear flow over a densely-packed sediment bed, 50 however, particle-particle contact becomes ubiquitous Balachandar and Eaton, 2010). Hard sphere models cannot maintain simultaneous collisions or enduring contacts between multiple interacting particles, but instead represent sediment beds as particles in constant, minute motion (Derksen, 2015). However, they have been used to reproduce critical erosion conditions for a laminar shear flow (Derksen, 2011). The drawbacks of the RP model for the situation of sediment transport have been clearly-elaborated by Kempe et al. (2014). Using the RP in the framework of the IBM introduces an artificial gap of two times the grid cell size between colliding particles so that the fluid in the gap between the particle surfaces can still be resolved (Glowinski et al. 2001). For the situation

60 of sediment transport, however, the artificial gap also introduces an unphysical protrusion of the particles into the horizontal flow, which is critical as the 
protrusion has been acknowledged to be a very sensitive parameter for particle mobilization (Fenton and Abbott, 1977). In addition, the RP model introduces a material stiffness $k_{n}$ which has to be calculated a priori to design a collision model that is numerically stable. If the value of $k_{n}$ is chosen too high, the repulsive force is overestimated and the particle would experience an unphysical high rebound velocity. On the other hand, if $k_{n}$ is too low, the duration of the particle collision would be too large. The resuspension mechanisms, however, generate a high variety of particle impacts velocities $u_{i n}$ for the collisions, typically characterized by the nondimensional Stokes number $\mathrm{St}=\rho_{p} u_{i n} D_{p} /\left(9 \rho_{f} \nu_{f}\right)$, where $\rho_{p}$ and $\rho_{f}$ are the particle and fluid density, respectively, $D_{p}$ is the particle diameter, and $\nu_{f}$ is the kinematic velocity of the fluid. In fact, for bed-load transport in water the variety can span from St $\gg 10$ (saltating particles, Niño and García, 1998) to $\mathrm{St}=\mathcal{O}(10)$ (rolling particles, Lajeunesse et al., 2010) to

75 St $\ll 1$ (enduring contact within the sediment bed). Hence, selecting a stiffness that is stable for high-impact velocities results in excessively low stiffnesses within the bed, which acts as an unphysical dampening of the system.

q Although there are studies of particle-laden horizontal flows such as Shao et al. (2012) and Kidanemariam et al. (2013) in which the model by Glowinski

80 et al. (1999) has been employed, these had to be limited to small volume fractions and conclusions about particle-particle interaction have not been possible. Kidanemariam and Uhlmann (2014) used an improved repulsive linear springdashpot model that solves the issues with calibrating $k_{n}$, but still relies on an artificial gap distance. Thus, in order to obtain appropriate bulk sediment transport quantities, they calibrated the dry restitution coefficient $e_{d r y}=-u_{\text {out }} / u_{\text {in }}$, where $u_{\text {out }}$ is the rebound velocity as soon as the collision process is finished, although this parameter can be set exactly as a material property. It describes the dissipation of kinetic energy due to the inelastic mechanics of the dry contact and is typically in the range of $0.8 \leq e_{d r y}<1$ for silicate materials (e.g.

90 Joseph et al. 2001). In the study of Kidanemariam and Uhlmann (2014), a rather unphysical value of $e_{d r y}=0.3$ was used to match the bulk transport rates of glass spheres from the experiments of Aussillous et al. (2013). 
More recently, a more consistent approach has been advocated in the litprature for which the artificial gap size is no longer needed Simeonov and 95 Calantoni, 2012, Kempe and Fröhlich, 2012a, Izard et al. 2014; Costa et al. 2015: Sierakowski and Prosperetti, 2016). This approach uses a lubrication force when the particles come in close contact $\left(0<\zeta_{n} \leq 2 h\right.$, where $\zeta_{n}$ is the distance between the two surfaces), and a contact force when the surfaces come in contact and slightly overlap $\left(\zeta_{n} \leq 0\right)$. The lubrication force, which is based on lubrication theory, models the fluid forces acting on the particle that cannot be resolved by the computational mesh. The contact force models material deformations and friction through components that are, respectively, normal and tangent to the surface. Since these models attempt to address the actual physics of the collision, they have had much success in reproducing the desired restitution coefficients over a range of Stokes numbers for the experiments by Joseph et al. (2001). Simeonov and Calantoni (2012) performed a detailed analysis breaking down the individual effects from lubrication forces, contact forces, and hydrodynamic forces. However, only Kempe and Fröhlich (2012a) and Costa et al. (2015) have demonstrated that their models were able to reproduce the trajectories of a particle-wall impact provided by the benchmark experiments of Gondret et al. (2002). Both studies show that the good agreement with the rebound trajectories is made possible by slightly stretching the collision process in time: long enough to resolve the response of the fluid field to the particle kinematics but shorter than any relevant physical timescale in the flow. In addition, both modelling approaches use an adaptive procedure to obtain mathematically-rigorous solutions to the ordinary differential equations governing particle motion during contact. Using the model of Kempe and Fröhlich (2012a), a breakthrough was achieved by Vowinckel et al. (2014), who successfully carried out numerical simulations of turbulent horizontal channel flow laden with tens of thousands of particles.

In the present work, we build on the model proposed by Kempe and Fröhlich 2012a to extend it to situations of very dense packing fractions. For example, in order for their collision model to work as designed, Kempe and Fröhlich 
2012a neglect the hydrodynamic forces acting on a particle while it is in contact more surprising, since its impact has been acknowledged as a key issue in the development and evolution of bedforms by segregation effects as reviewed by Charru et al. (2013). 
As a consequence, the present work aims to resolve the problems mentioned above. Among the key challenges identified are i) deriving collision models for polydisperse sediment, ii) avoiding the introduction of an artificial gap between colliding particles, iii) adaptively-calibrating the particle stiffness to simulate a wide range of Stokes numbers in a consistent manner, iv) introducing suitable criteria to extend existing models towards the numerically-challenging situation of enduring contact for both normal and oblique collisions, and v) minimizing the number of tunable parameters within the model framework. We achieve our goals by presenting an implementation of collision models for polydisperse sediment. We use the adaptive procedure proposed by Kempe and Fröhlich (2012a) for normal forces and the tangential model of Thornton et al. (2013), which stems from DEM. Furthermore, we extend both of these approaches for the situation of enduring contact. In particular, for enduring contact, we took care to retain all the governing terms of the momentum balance of a particle, i.e. hydrodynamic forces, buoyant weight, and collisions forces. This measure turns out to be crucial when simulating flows over sediment beds, as the Shields parameter is based on the ratio of hydrodynamic to buoyant forces. The proposed enhancements allow us to reproduce several laboratory benchmark test cases for binary collisions. In addition, we present a detailed validation of our simulation results with wall-normal profiles of the fluid and particle velocities 175 as well as bulk flow quantities using the experimental data of Aussillous et al. (2013),

The paper is structured as follows. We briefly recall the numerical method including fluid solver, IBM, and the structure of the collision model in Section 2 , followed by the mathematical description of the collision model employed in Section 3. We then present necessary enhancements to the collision model to deal with small Stokes numbers (Section 4) and to simulate dense granular packings with the gross of the particles in enduring contact (Section 5). Subsequently, the enhanced model is validated for the collective motion of polydisperse sediment sheared by a laminar flow in Section 6 . 


\section{Particle motion and four-way coupling in the framework of the Immersed Boundary Method}

\subsection{Fluid solver}

For the present simulations, we solve the unsteady Navier-Stokes equations for an incompressible Newtonian fluid, given by

$$
\frac{\partial \mathbf{u}}{\partial t}+\nabla \cdot(\mathbf{u u})=-\frac{1}{\rho_{f}} \nabla p+\nu_{f} \nabla^{2} \mathbf{u}+\mathbf{f}_{I B M},
$$

and the continuity equation, given by

$$
\nabla \cdot \mathbf{u}=0
$$
using second-order central-differencing. The pressure is treated with a direct solver based on Fast Fourier Transformations (FFT). Our code can handle a 
variety of boundary conditions at the different walls, including no-slip, slip, periodic, and inflow/outflow.

\subsection{Computation of particle motion}

Within the framework of the IBM, we calculate the motion of each individual spherical particle by solving an ordinary differential equation for its translational velocity $\mathbf{u}_{p}=\left(u_{p}, v_{p}, w_{p}\right)^{T}$

$$
m_{p} \frac{\mathrm{d} \mathbf{u}_{p}}{\mathrm{~d} t}=\underbrace{\oint_{\Gamma_{p}} \boldsymbol{\tau} \cdot \mathbf{n} \mathrm{d} A}_{=\mathbf{F}_{h, p}}+\underbrace{V_{p}\left(\rho_{p}-\rho_{f}\right) \mathbf{g}}_{=\mathbf{F}_{g, p}}+\mathbf{F}_{c, p}
$$

and its angular velocity $\boldsymbol{\omega}_{p}=\left(\omega_{p, x}, \omega_{p, y}, \omega_{p, z}\right)^{T}$

$$
I_{p} \frac{\mathrm{d} \boldsymbol{\omega}_{p}}{\mathrm{~d} t}=\underbrace{\oint_{\Gamma_{p}} \mathbf{r} \times(\boldsymbol{\tau} \cdot \mathbf{n}) \mathrm{d} A}_{=\mathbf{T}_{h, p}}+\mathbf{T}_{c, p}
$$

Here, $m_{p}$ is the particle mass, $\Gamma_{p}$ the fluid-particle interface, $\boldsymbol{\tau}$ the hydrodynamic stress tensor, $\rho_{p}$ the particle density, $V_{p}$ the particle volume, $g$ the gravitational acceleration, $I_{p}=8 \pi \rho_{p} R_{p}^{5} / 15$ the moment of inertia, and $R_{p}$ the particle radius. Furthermore, the vector $\mathbf{n}$ is the outward-pointing normal on the interface $\Gamma_{p}$, $\mathbf{r}=\mathbf{x}-\mathbf{x}_{p}$ is the position vector of the surface point with respect to the center of mass $\mathbf{x}_{p}$ of a particle, and $\mathbf{F}_{c, p}$ and $\mathbf{T}_{c, p}$ are the force and torque due to particle collisions, respectively. Furthermore, note the designation of the hydrodynamic force and torque as $\mathbf{F}_{h, p}$ and $\mathbf{T}_{h, p}$, respectively, as well as $\mathbf{F}_{g, p}$ the force due to gravity, which will be used in the following for brevity.

We employ the approach of Kempe and Fröhlich (2012b) for evaluating the IBM forces and solving (3) and (4). We validated the fluid-particle coupling of the method against experimental data of a sphere settling in an unbounded quiescent fluid (Mordant and Pinton, 2000) as well as towards a wall (Ten Cate et al., 2002). The particle was initially at rest for both setups and accelerated downwards due to gravity. For the unbounded case, the particle reached a constant terminal velocity $u_{\infty}$. The Reynolds number $\operatorname{Re}_{p}=u_{\infty} D_{p} / \nu_{f}$ based on this settling velocity is $\operatorname{Re}_{p}=41$. For the wall-bounded case, the particle 

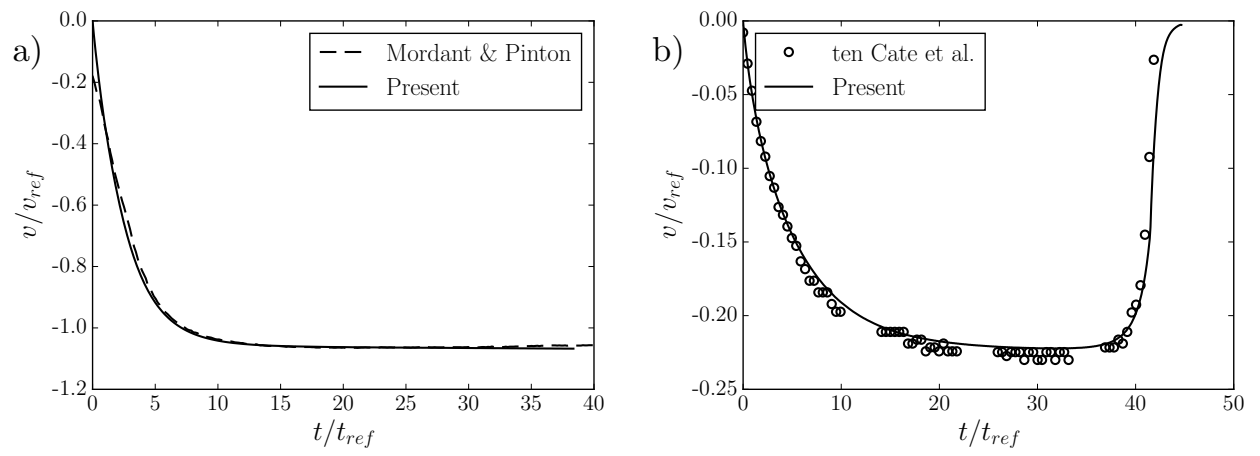

Figure 1: Comparison of the present numerical method against experimental data with $v_{r e f}=\sqrt{g D_{p}}$ and $t_{r e f}=\sqrt{D_{p} / g}$. a) Settling sphere in an infinite medium at $\mathrm{Re}_{p}=41$ (Mordant and Pinton, 2000) and b) settling sphere in a wall-bounded medium at $\operatorname{Re}_{p}=12$ (Ten Cate et al., 2002).

reached a terminal velocity but then decelerated as it approached the wall. For now, we only consider the particle motion before impact, which is governed by the IBM. The collision model governing the impact will be described and validated further below in the text. The Reynolds number based on the velocity before deceleration is $\operatorname{Re}_{p}=12$. In both cases, the particle was discretized with 20 grid cells per diameter. The respective data are plotted in Figure 1 , showing excellent agreement.

\subsection{Structure of the collision model}

As mentioned in the introduction, one of the major advantages of the IBM is the direct computation of long-range interactions between the particles. Only short-range interactions and collisions need to be modeled. For example, consider a particle approaching and colliding with a wall, as shown in Figure2, As the particle comes close to the wall, two problems need to be dealt with: first, the smoothed Dirac delta functions used for the IBM overlap with the wall, and second, the discrete mesh can no longer resolve the fluid being squeezed out from between the two surfaces.

We can solve the first problem by disabling Lagrangian marker points whose 

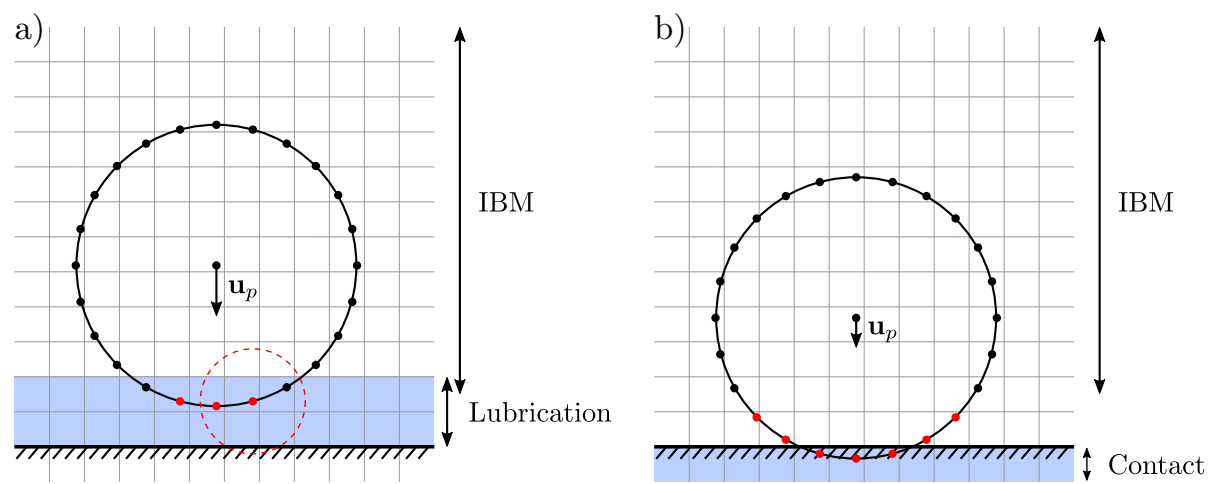

Figure 2: Regions where short-range interactions occur. Points on particle surface represent Lagrangian markers. Red markers have been turned off. a) Lubrication region where red dashed circle illustrates support of Dirac delta function and b) Contact region.

supports overlap with the wall (red dashed circle in Figure 22), as was done by Kempe and Fröhlich (2012b). This means that the forcing by these select markers on both the fluid and the particles is ignored, preventing the particle from using undefined information from outside of the domain and from competing with the wall for enforcing the no-slip condition. Figure 2 illustrates the red markers that have been disabled. Similarly, overlapping markers between two particles are disabled. We solve the second problem by adding a lubrication force, which models the subgrid forces on the particle due to the narrow gap and also accounts for some of the fluid forces from the disabled Lagrangian markers. We apply this force when the particle-wall distance is less than two grid cells $\left(0<\zeta_{n} \leq 2 h\right)$, illustrated by the blue region in Figure 2 a. Once the particle comes into contact with the wall $\left(\zeta_{n} \leq 0\right)$, we apply a contact force to prevent particles from overlapping too much and to account for proper momentum transfer and energy loss. This contact force involves components both normal and tangent to the two surfaces, representing material deformations and friction, respectively.

Hence, the following case distinctions can be made for the normal collision 


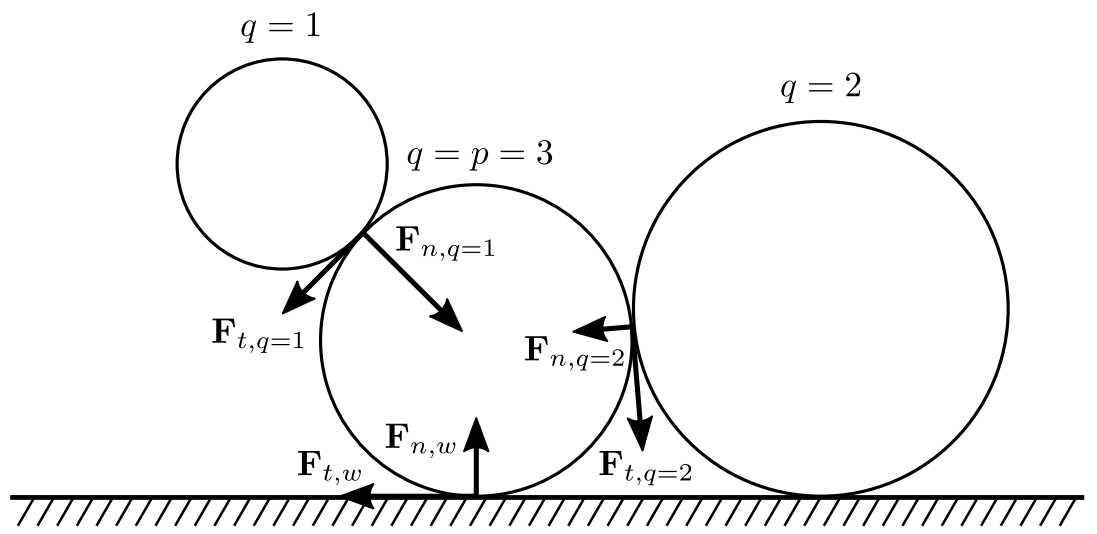

Figure 3: Sketch of polydisperse particles in a mobile granular bed and the resulting interactions due to collisions.

forces

$$
\mathbf{F}_{n}= \begin{cases}0 & \zeta_{n}>2 h \\ \text { lubrication model }(9) & 0<\zeta_{n} \leq 2 h \\ \text { normal contact model 10] } & \zeta_{n} \leq 0\end{cases}
$$

and the tangential collision forces

$$
\mathbf{F}_{t}=\left\{\begin{array}{ll}
0 & \zeta_{n}>0 \\
\text { tangential contact model } & \zeta_{n} \leq 0
\end{array} .\right.
$$

The interactions of a single particle with its environment, however, are in general more diverse. Let us consider a particle $p$ embedded in a mobile granular bed of polydisperse, spherical particles (Figure 3). The dynamics of this particle are mainly determined by all the collision forces exerted upon it by particles $q, q \neq p$ as well as the wall. The total force $\mathbf{F}_{c, p}$ acting on a particle $p$ during the collision process may be decomposed as

$$
\mathbf{F}_{c, p}=\sum_{q, q \neq p}^{N_{p}}\left(\mathbf{F}_{n, q}+\mathbf{F}_{t, q}\right)+\mathbf{F}_{n, w}+\mathbf{F}_{t, w}
$$

280

where $N_{p}$ is the number of particles simulated, $\mathbf{F}_{n, q}$ and $\mathbf{F}_{n, w}$ are the normal collision forces described by (5) with particle $q$ and the wall, respectively, and 
$\mathbf{F}_{t, q}$ and $\mathbf{F}_{t, w}$ are the tangential collision forces described by (6) with particle $q$ and the wall, respectively. In what follows, the mathematical expressions are formulated for both particle-wall and particle-particle collisions, where the radii of the two colliding particles can be arbitrary. Whenever a distinction between particle-wall and particle-particle has to be made, a reference to Appendix A is given for brevity, providing all definitions and nomenclature needed to distinguish between the two different cases.

The torque $\mathbf{T}_{c, p}$ on a spherical particle $p$ generated by the tangential contact

$$
\mathbf{T}_{c, p}=\sum_{q, q \neq p}^{N_{p}} R_{p, c p} \mathbf{n}_{p, q} \times \mathbf{F}_{t, q}+R_{p, c p} \mathbf{n}_{p, w} \times \mathbf{F}_{t, w}
$$

where $\mathbf{n}_{p, q}$ and $\mathbf{n}_{p, w}$ are the unit vectors pointing to the collision partner $q$ or the wall, respectively, and $R_{p, c p}$ is the particle radius at the contact point as defined per Appendix A in A.6), which accounts for surface overlap. In the next section, we will provide the mathematical description of the models used in the present study.

\section{Collision modeling}

\subsection{Lubrication model}

When the distance between the surfaces of two approaching particles becomes small, the fluid is squeezed out of the gap. The fluid grid cannot resolve this process as soon as $\zeta_{n}<2 h$, where $h$ is the grid cell size. Hence, we employ a lubrication model, which also acts on particles rebounding after the collision, when fluid is drawn into the gap. The lubrication force is dissipative, since it is always directed opposite to the relative velocity. The model is based on the analytical derivation of Cox and Brenner (1967), who solved for the force under Stokes flow conditions

$$
\mathbf{F}_{n}=-\frac{6 \pi \rho_{f} \nu_{f} R_{e f f}^{2}}{\max \left(\zeta_{n}, \zeta_{n, \min }\right)} \mathbf{g}_{n, c p}
$$

where $R_{e f f}$ is the effective radius accounting for polydisperse sediment and $\mathbf{g}_{n, c p}$ the normal component of the relative particle velocity as defined per Appendix 
310 introduces a singularity as $\zeta_{n} \rightarrow 0$. This has been addressed by Simeonov and Calantoni (2012) and Kempe and Fröhlich (2012a), who set $\mathbf{F}_{n}=0$ for $0<\zeta_{n}<\zeta_{n, \min }$, and Izard et al. (2014), who have shifted the denominator of (9) to $\zeta_{n}+\zeta_{n, \min }$. In the present approach, as in the approach of Costa et al. Fröhlich, 2012a: Costa et al. 2015). However, the duration of contact $T_{c}$ is mainly determined by the stiffness parameter $k_{n}$. This becomes of particular importance for the complex situation of bed-load transport, where a broad range

which involves empirical parameters for the coefficients of stiffness $k_{n}$ and damping $d_{n}$. Here, $\mathbf{n}$ is the normal vector pointing either towards the collision partner or towards the wall as defined per Appendix A in A.3. The nonlinear term $\left|\zeta_{n}\right|^{3 / 2}$ arises from Hertzian contact theory (Hertz, 1882).

Since the timescale of a collision according to Hertzian contact theory is several orders of magnitude smaller than the typical temporal discretization of the fluid solver, the collision event has to be stretched in time to maintain the efficiency of the numerical procedure. This measure is also needed for the fluid to adapt to the sudden change in the particle trajectory Kempe and 
of impact velocities is encountered, ranging from high-impact collisions at the top of the bed to enduring contact within the bed.

The ACTM fixes this problem by adaptively calibrating the parameters $k_{n}$ and $d_{n}$ depending on the impact velocity $u_{i n}$, the desired restitution coefficient $e_{d r y}$, and the desired collision time $T_{c}$. The latter is a parameter of the model and should be minimized to avoid excessive particle overlaps and temporal stretching. Kempe and Fröhlich (2012a) demonstrated that $T_{c}=10 \Delta t$ is a suitable choice for the collision time given that all timescales related to fluid and particle motion are significantly larger than the timescale of particle contact. For glass and hard metals, $e_{d r y}=0.97$ is a typical value (e.g Foerster et al. 1994, Joseph et al., 2001; Gondret et al., 2002). For immersed collisions, the 350 restitution coefficient $e_{w e t}$, measured some small distance away from the wall, becomes a function of the Stokes number (Joseph et al. 2001). The ACTM, however, uses the IBM and lubrication model to account for $e_{w e t}$ through additional dissipative fluid effects.

In order to find values for $k_{n}$ and $d_{n}$, we first neglect all non-contact forces acting on the particle so that $(3)$ and $\sqrt{10}$ together give the following nonlinear ordinary differential equation:

$$
m_{e f f} \frac{\mathrm{d}^{2} \zeta_{n}}{\mathrm{~d} t^{2}}+d_{n} \frac{\mathrm{d} \zeta_{n}}{\mathrm{~d} t}+k_{n} \zeta_{n}^{3 / 2}=0
$$

where $m_{\text {eff }}$ is the effective mass accounting for polydisperse sediment as defined per Appendix A in A.2. Note that $\mathrm{d} \zeta_{n} / \mathrm{d} t=-\mathbf{g}_{n, c p} \cdot \mathbf{n}$. Together with (11) and initial and final conditions, the constraints $e_{d r y}$ and $T_{c}$ allow for determination of $k_{n}$ and $d_{n}$ using either an iterative procedure, as was done by Kempe and Fröhlich (2012a), or an explicit formulation, as was proposed by Ray et al. (2015). In the present study, we implemented the explicit formulation, which is provided in Appendix B. According to Ray et al. (2015), the error in $u_{\text {out }}$ increases with decreasing $e_{d r y}$, but does not exceed $1.3 \%$ for $e_{d r y}>0.7$ or $3 \%$ for $e_{d r y}>0.4$, making this method useful for most sediment materials such as silicate, glass, or even metal. 


\subsection{Tangential collision model}

To account for frictional contact between the particles, we implemented a the review paper of Thornton et al. (2013):

$$
\mathbf{F}_{t, L S}=-k_{t} \boldsymbol{\zeta}_{t}-d_{t} \mathbf{g}_{t, c p}
$$

which has stiffness and damping coefficients $k_{t}$ and $d_{t}$. This model uses $\mathbf{g}_{t, c p}$, the tangential component of the relative surface velocities as described in A.10 of

375 Appendix A as well as $\boldsymbol{\zeta}_{t}$, the tangential spring displacement, which represents the accumulated relative tangential motion between the two surfaces:

$$
\boldsymbol{\zeta}_{t}=\int_{t_{i}}^{t} \mathbf{g}_{t, c p}\left(t^{\prime}\right) d t^{\prime}
$$

where $t_{i}$ is the time of impact. The discretized form of $\sqrt{13}$ is described in Appendix C

This model limits the maximum force based on Coulomb's friction criterion:

$$
\mathbf{F}_{t}=\min \left(\left\|\mathbf{F}_{t, L S}\right\|,\left\|\mu \mathbf{F}_{n}\right\|\right) \mathbf{t} \quad,
$$

where $\mu$ represents the coefficient of friction between the two surfaces (described further in Section 5.3 and $\mathbf{t}=\mathbf{F}_{t, L S} /\left\|\mathbf{F}_{t, L S}\right\|$ points in the direction of the tangential force.

This model has two important features for simulating densely-packed beds. First, the spring allows many particles to interact in a smooth, stable manner, provided the stiffness is chosen properly. Second, the model has a memory of the friction force via the tangential displacement $\boldsymbol{\zeta}_{t}$, which permits a steady-state frictional bed configuration. In contrast, a model that only uses $\mathbf{g}_{t, c p}$, such as the one proposed by Kempe and Fröhlich (2012a), can only react to slip, not predict it.

Similarly to the ACTM, we can adaptively compute $k_{t}$ and $d_{t}$ for each collision. According to Thornton et al. (2011), the stiffness can be set to

$$
k_{t}=\frac{\kappa m_{\text {eff }} \pi^{2}}{T_{c}^{2}}
$$


Here, $\kappa$ is based on Poisson's ratio $\nu$ :

$$
\kappa=\frac{2(1-\nu)}{2-\nu}
$$

which is a well-studied material property typically ranging between $0.22<\nu<$ 0.30 (e.g Foerster et al., 1994, Gondret et al., 2002, Joseph and Hunt, 2004). Hence, a value of $\nu=0.22$ was used in the present study.

In addition, the damping is computed according to Thornton et al. (2013) to account for the inelasticity of the collisions

$$
d_{t}=2 \sqrt{m_{e f f} k_{t}} \frac{-\ln e_{d r y}}{\sqrt{\pi^{2}+\ln ^{2} e_{d r y}}} .
$$

Having created a uniform collision time $T_{c}$ with the normal contact model, we obtain the correct rebound characteristics for oblique impacts using these values for $k_{t}$ and $d_{t}$, as shown in Section 5.3. Consistently, the model does not require any calibration but instead can be parameterized using material properties obtained from experiments.

\section{Enhancements to the normal contact model}

\subsection{Motivation}

In order to obtain a good agreement with immersed collision experiments, we had to implement a few enhancements to the normal contact model described in Section 3.2. Both changing the time integration to a scheme of higher accuracy and adding more timesteps to the integration of particle motion without changing the fluid timestep allowed us to reproduce the collision trajectories of Gondret et al. (2002) in a robust manner.

\subsection{Improved time integration}

The ACTM normal contact force $\mathbf{F}_{c, p}$ is a function of the surface distance $\zeta_{n}$ and the relative velocity $\mathbf{g}_{n, c p}$, which in turn depend on the particle position $\boldsymbol{x}_{p}^{k-1}$ and velocity $\boldsymbol{u}_{p}^{k-1}$ at the previous substep $k-1$. We can write 
this functional dependence as $\mathbf{F}_{c, p}\left(\mathbf{x}_{p}^{k-1}, \mathbf{u}_{p}^{k-1}\right)$. Integrating the particle equation of motion with a Forward Euler/Crank Nicholson scheme for the particle's velocity/position, we obtain:

$$
\begin{aligned}
& \mathbf{u}_{p}^{k}=\mathbf{u}_{p}^{k-1}+\frac{2 \Delta t \alpha_{k}}{m_{p}} \mathbf{F}_{c, p}\left(\mathbf{x}_{p}^{k-1}, \mathbf{u}_{p}^{k-1}\right) \\
& \mathbf{x}_{p}^{k}=\mathbf{x}_{p}^{k-1}+\Delta t \alpha_{k}\left(\mathbf{u}_{p}^{k}+\mathbf{u}_{p}^{k-1}\right)
\end{aligned}
$$

¡where $k$ is the number of the RK-substep and $\alpha_{k}$ is the RK-coefficient (Rai and Moin, 1991). For now, we ignore the hydrodynamic, gravitational, and lubrication forces in order to focus on the contact forces alone. We conducted a simple test to analyze the accuracy of this scheme. A particle of density $\rho_{p} / \rho_{f}=7.8$ and with radius $R_{p}=10 u_{\infty} \Delta t$ was initialized with a velocity of $\mathbf{u}_{p}=\left(0, u_{\infty}, 0\right)^{T}$ at a position $y_{p}>R_{p}$ above a wall at $y=0$. Subsequently, the particle was released and eventually collided with the wall. Neglecting hydrodynamic effects as well as gravity yields an impact velocity of $u_{i n} / u_{\infty}=1$. Choosing the collision time to be $T_{c}=10 \Delta t$ as suggested by Kempe and Fröhlich (2012a), gave good results for the duration of the desired contact phase $T_{c}$, but rather large errors of the rebound velocity were observed compared to the prescribed $e_{d r y}=1$. The value of $u_{\text {out }}=-e_{d r y} u_{i n}$ was overestimated by more than $12 \%$.

Turning our attention to Figure 4, we can see that the discretization of (18) leads to a poor estimation of the collision force $\left\|\mathbf{F}_{c, p}\right\|$ when compared to the simulation in which $10^{4}$ timesteps were used to resolve the collision, which can be taken as the exact solution. This inaccuracy in the collision force was observed for a variety of simulations using different $R_{p}, \rho_{p}, u_{i n}$, and $e_{d r y}$. In order to reduce the error to $0.1 \%$, a temporal discretization of $T_{c}=1000 \Delta t$ would be required, which is not feasible for simulations of sediment transport. Hence, we implemented a temporal discretization scheme with a higher order of accuracy. Utilizing the same three-step RK scheme that integrates the NavierStokes equations, we reformulated the collision integration with a predictor- 
corrector scheme:

$$
\begin{aligned}
\widetilde{\mathbf{u}}_{p} & =\mathbf{u}_{p}^{k-1}+\frac{\Delta t}{m_{p}}\left[\gamma_{k} \mathbf{F}_{c, p}\left(\mathbf{x}_{p}^{k-1}, \mathbf{u}_{p}^{k-1}\right)+\zeta_{k} \mathbf{F}_{c, p}\left(\mathbf{x}_{p}^{k-2}, \mathbf{u}_{p}^{k-2}\right)\right] \\
\widetilde{\mathbf{x}}_{p} & =\mathbf{x}_{p}^{k-1}+\Delta t \alpha_{k}\left(\widetilde{\mathbf{u}}_{p}+\mathbf{u}_{p}^{k-1}\right) \\
\mathbf{u}_{p}^{k} & =\mathbf{u}_{p}^{k-1}+\frac{\Delta t \alpha_{k}}{m_{p}}\left[\mathbf{F}_{c, p}\left(\widetilde{\mathbf{x}}_{p}, \widetilde{\mathbf{u}}_{p}\right)+\mathbf{F}_{c, p}\left(\mathbf{x}_{p}^{k-1}, \mathbf{u}_{p}^{k-1}\right)\right] \\
\mathbf{x}_{p}^{k} & =\mathbf{x}_{p}^{k-1}+\Delta t \alpha_{k}\left(\mathbf{u}_{p}^{k}+\mathbf{u}_{p}^{k-1}\right)
\end{aligned}
$$

\subsection{Temporal substepping}

Having improved the accuracy of the contact model, we carried out simulations of particle-wall collisions in a fluid to compare to the experiments of Gondret et al. (2002). The details of the simulations, including the material properties as well as the physical and numerical parameters, are summarized in Table 1. Gondret et al. (2002) released particles from heights large enough to accelerate to their terminal velocities before colliding with the wall. For these simulations, the horizontal wall and vertical particle trajectories allow us to

only consider normal collision forces. To control the impact velocity $u_{i n}$, we accelerated the particle in the numerical simulations according to the relation

$$
u(t)=u_{i n}\left(e^{-40 t}-1\right), \quad \zeta_{n}>R_{p}
$$




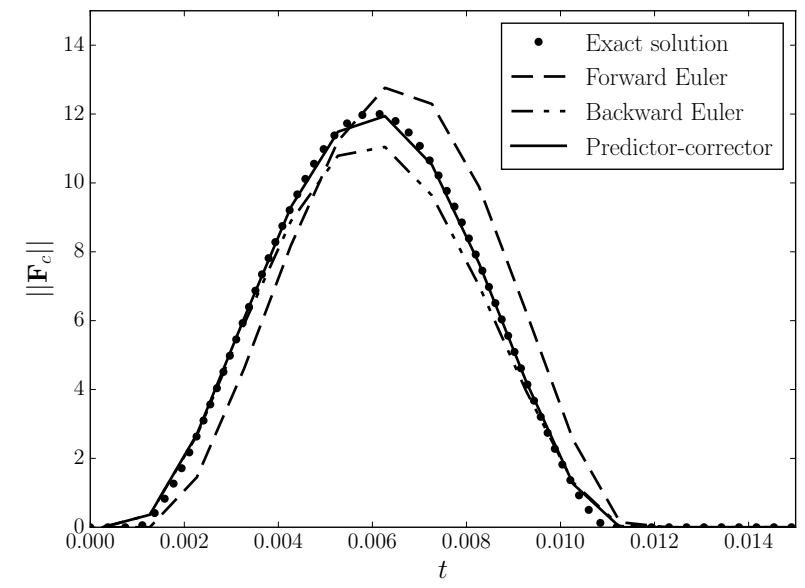

Figure 4: Collision forces vs. time for Forward Euler, Backward Euler, and predictorcorrector schemes.

In other words, we prescribed the falling velocity of the particle so that it accelerated in a smooth manner so that $u_{i n}$ matched the Stokes number reported in Gondret et al. (2002) as shown in Table 1. Two scenarios were considered: one with a rather high Stokes number St $=152$ and one with a lower Stokes number of $\mathrm{St}=27$, the latter of which is within the range of Stokes numbers that have been reported for the numerical simulations of Kempe et al. (2014). Once the particle reached a distance of $\zeta_{n}=R_{p}$, we turned off the prescribed velocity, allowing the particle to move on its own volition according to the hydrodynamic, buoyant, and collision forces acting on it.

While attempting to reproduce the experimental trajectories, the simulations produced large variations in the results from small changes to the initial conditions. To show this, we executed five simulations for $\mathrm{St}=27$, varying only the initial position of the particle $y_{0}$ from the value recorded in Table 1 within the interval of one grid cell $h$. Figure 5 a shows the range of trajectories encountered. For $\mathrm{St}=27$, a substantial variation in the rebound height of up to $83 \%$ can be observed. We would thus expect the collision model to produce 460 a variety of incorrect trajectories for the simulation of sediment transport in a horizontal channel flow. Even the mean of the variety of trajectories is not able 


\begin{tabular}{lcc}
\hline \hline $\mathrm{St}$ & 27 & 152 \\
$\mathrm{Re}_{p}$ & 30 & 164 \\
\hline$R_{p}(\mathrm{~m})$ & 0.003 & 0.0015 \\
$u_{i n}(\mathrm{~m} / \mathrm{s})$ & 0.518 & 0.585 \\
$\rho_{p} / \rho_{f}$ & 8.083 & 8.342 \\
$\nu_{f}\left(\mathrm{~m}^{2} / \mathrm{s}\right)$ & $1.036 \times 10^{-4}$ & $1.070 \times 10^{-5}$ \\
$e_{d r y}$ & 0.97 & 0.97 \\
$g\left(\mathrm{~m} / \mathrm{s}^{2}\right)$ & 9.81 & 9.81 \\
\hline Domain size $(\mathrm{m})\left(L_{x} \times L_{y} \times L_{z}\right)$ & $0.08 \times 0.16 \times 0.08$ & $0.02 \times 0.2 \times 0.02$ \\
Domain boundary conditions & $\mathrm{p} \times \mathrm{ns} \times \mathrm{p}$ & $\mathrm{p} \times \mathrm{ns} \times \mathrm{p}$ \\
Initial position of sphere center $(\mathrm{m})$ & 0.075 & 0.197 \\
Grid cells in $x$-direction & 256 & 128 \\
Grid cells per diameter & 19 & 19 \\
Timestep & $\Delta t=2.5 \mathrm{e}-4$ & $\Delta t=8.9 \mathrm{e}-5$ \\
\hline \hline
\end{tabular}

Table 1: Simulation parameters to match the experiments of Gondret et al. (2002). Boundary conditions can be periodic (p), slip (s), or no-slip (ns).

to fully reproduce the experimental trajectory.

To better understand the observed variability, we plot the time evolution of the collision forces, i.e. lubrication and contact forces, for the low Stokes number case $\mathrm{St}=27$ in Figure 6. In this plot, we can see the particle approaching the wall with the lubrication force growing as $1 / \zeta_{n}$ (phase I). Subsequently, the lubrication forces become zero during the contact phase starting at $t / t_{r e f}=-0.1$. During this phase (phase II), the contact force grows and then decays with the particle-wall overlap as the particle changes direction to rebound. Finally, the particle experiences the lubrication force again during the rebound phase starting at $t / t_{r e f}=0$ (phase III). At this time, lubrication is acting in the opposite direction because lubrication is dissipative. The dotted line in Figure 6 shows the forces acting on the particle for a time discretization based on CFL $=0.5$ for the settling velocity. As expected, the normal contact model with the mod- 

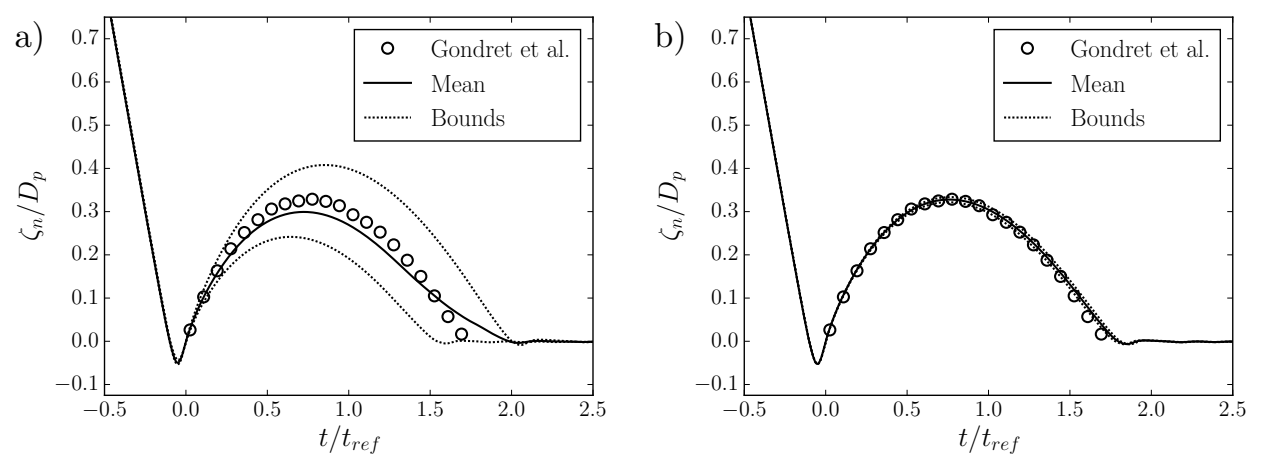

Figure 5: Sensitivity of rebound trajectories to initial position $y_{0}$ for $\mathrm{St}=27$. a) Trajectories computed without particle substeps and b) trajectories computed with particle substeps.

ifications described in Section 4.2 above is able to give a smooth evolution of contact forces with the time step size of the fluid solver. However, it turns out that the lubrication forces remain under-resolved during approach and rebound, especially as $\zeta_{n}$ approaches zero directly before and after the contact phase. This leads to either more or less total impulse acting on the particle, depending on where the timestep happens to land, which in turn results in variability between simulations. This effect strongly depends on the Stokes number, since the lubrication force decreases with increasing St. Hence, the ratio of the normal contact force to the lubrication force also decreases when approaching dry contact conditions.

Since the lubrication model used is an algebraic relation that does not depend on the surrounding hydrodynamics, we have implemented a substepping method that integrates the particle motion with smaller timesteps than the fluid motion. This method works as follows:

1. We solve the fluid equations of motion, IBM, and hydrodynamic forces 490 acting on the particle as normal.

2. We divide the fluid RK substep $k$ into a number of substeps $N_{s u b, k}=$ $\{8,2,5\}$. This choice results in a total of 15 substeps of constant size per fluid timestep $\left(\Delta t_{s u b}=\Delta t / 15\right)$, which is most efficient since $2 \alpha_{k}=$ 


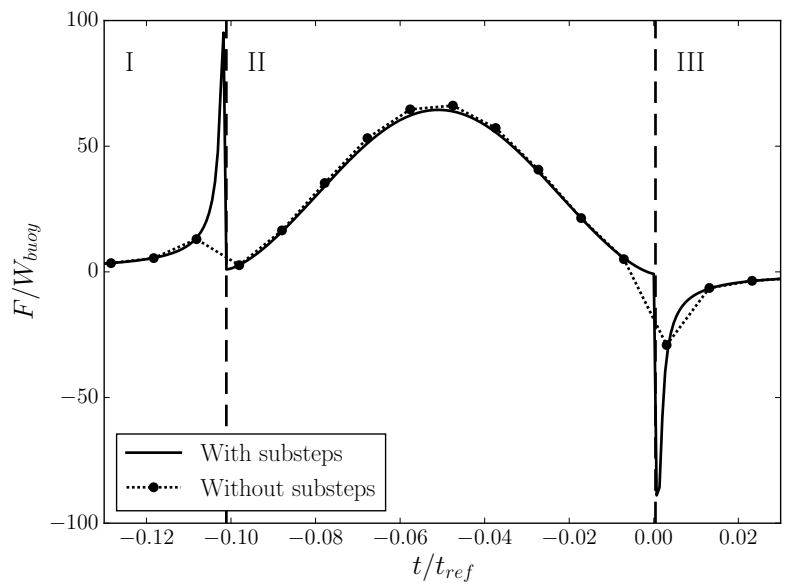

Figure 6: Collision forces acting on the particle at $\mathrm{St}=27$ including: the lubrication force during approach (phase I), the normal force during contact (phase II), and the lubrication force during rebound (phase III). Vertical dashed lines indicate a change in phase. $W_{\text {buoy }}=\left(1-\rho_{f} / \rho_{p}\right) m_{p} g$ is the buoyant weight of the particle.

$\{8 / 15,2 / 15,5 / 15\}$ as used in 19 .

3. For each of the substeps, we solve the particle equations of motion with the three-step RK method. As we update the particle velocities and positions, we re-evaluate the collision (lubrication and contact) forces, but the hydrodynamic forces remain constant. This compromise makes the present approach very efficient.

4. At the end of the 8,2 , or 5 substeps, we use the final particle position and velocity for the next fluid RK substep.

This measure effectively increases the resolution of a collision to a timestep 15 times smaller than the fluid timestep to integrate particle motion, allowing us to compute the lubrication forces with higher accuracy. Since the contact duration of $T_{c}=10 \Delta t$ is maintained, the contact phase is now resolved with a total of 150 timesteps with only a marginal increase to the computational cost. Substepping has also been used by Kidanemariam and Uhlmann (2014) and Costa et al. (2015) but the authors did not illustrate the variability we have observed for the trajectories of particle-wall collisions. Meanwhile, Kidanemariam and Uhlmann 
The scheme presented by Costa et al. (2015) still relies on an iterative procedure subdividing every fluid timestep into 50 substeps, which is less efficient than the scheme presented here. The results of our approach can be appreciated in Figure 6. The solid line, which was resolved with fifteen times more timesteps, can be viewed as a better approximation of the exact solution to the model we have implemented. Figure 5 b shows how this method almost eliminates the variability in the rebound trajectories of the particle-wall collisions discussed above.

\subsection{Choice of particle surface roughness}

As shown in Figure 5 the improved integration scheme described in Sections 4.2 and 4.3 yields excellent results in reproducing the rebound trajectory of the $\mathrm{St}=27$ experiment of Gondret et al. (2002). Having obtained consistent results that are insensitive to the initial condition, we can use the same setup of particle-wall collisions to select the most suitable surface roughness $\zeta_{n, m i n}$ for the lubrication model (9). This is the only parameter involved that requires calibration as an inverse problem. However, the range of values that can be assigned to $\zeta_{n, \min }$ should neither fall below the surface roughness of the actual simulated particle nor exceed the length of a grid cell in order for the lubrication model to make physical sense.

The impact of $\zeta_{n, \min }$ on particle rebound trajectories for $\mathrm{St}=27$ is illustrated in Figure 7. A clear trend can be identified: decreasing the value of $\zeta_{n, \min }$ also decreases the rebound height due to more damping within the lubrication layer. However, the results are moderately sensitive to the roughness value. For instance, note that changing the roughness by an order of magnitude present results, we selected $\zeta_{n, \min }=3 \mathrm{e}-3 R_{p}$ to optimize agreement with the experimental data. We have used this value for all simulations in the present ¡work. Note that a surface roughness of $1 \mathrm{e}-4 R_{p}$ has been reported by Gondret et al. (2002), and other authors have used the physical particle roughness length 


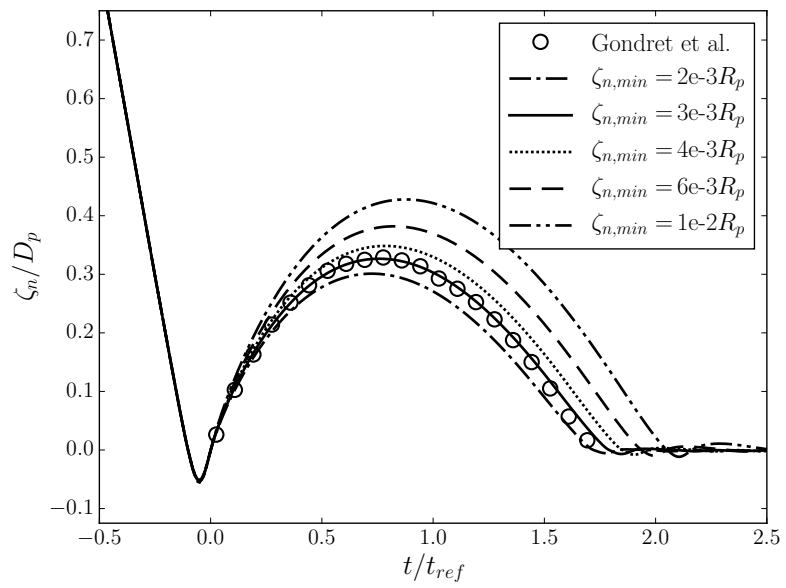

Figure 7: Effect of changing $\zeta_{n, \text { min }}$ on rebound trajectories for $\mathrm{St}=27$.

540 for this parameter (Kempe and Fröhlich, 2012a, Costa et al. 2015) to avoid the singularity in the lubrication force. Thus, we do not consider this parameter to be an exact physical representation of the actual surface roughness, but rather as a parameter to be calibrated within a reasonable range (small enough to be meaningful relative to the particle size and large enough to be resolved by the substeps).

\subsection{Particle momentum balance for high Stokes number collisions}

口 Finally, we present a clarification to the ACTM as written by Kempe and Fröhlich (2012a). As already mentioned in Section 3.2, the ACTM assumes that (11) represents the equation of motion for the particle in determining the coefficients $k_{n}$ and $d_{n}$. In other words, no fluid or gravitational forces act on the particle during the contact phase. Though not stated in their paper, Kempe and Fröhlich (2012a) excluded hydrodynamic and buoyant weight forces in order to reproduce the trajectories of Gondret et al. (2002) (Kempe \& Fröhlich, 2016, private communication). Thus, during contact the non-disabled Lagrangian markers still affect the fluid, but not the particle momentum. This procedure is somewhat delicate for the situation of sediment transport in a horizontal channel considering the fact that the governing nondimensional number is the ratio of the 

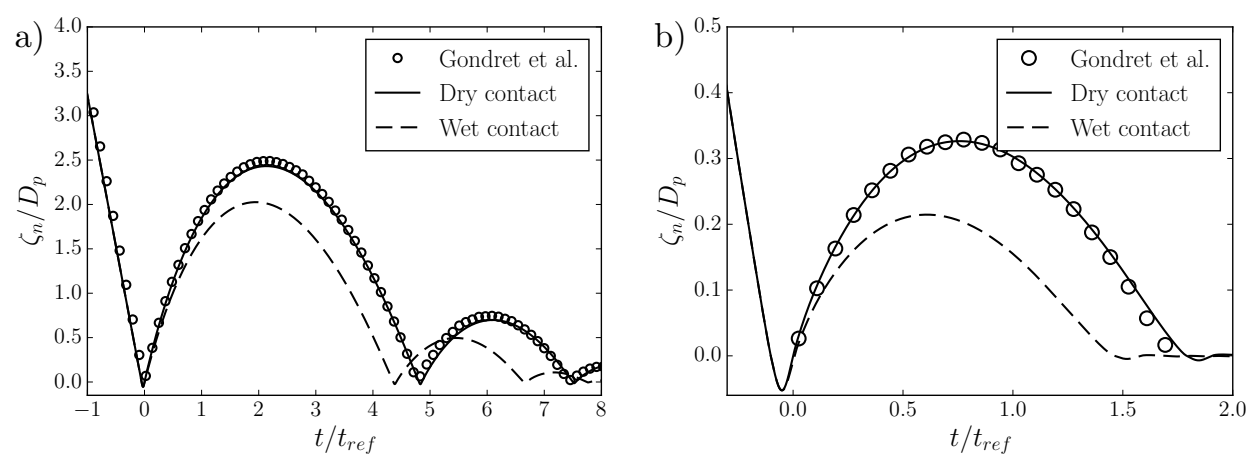

Figure 8: Effect of including ("wet") or excluding ("dry") fluid forces during contact on rebound trajectories. a) $\mathrm{St}=152$ and b) $\mathrm{St}=27$.

hydrodynamic stress to the buoyant weight of the particle. This characteristic number is classically known as the Shields parameter $\mathrm{Sh}=\tau_{w} /\left(\left(\rho_{p}-\rho_{f}\right) g D_{p}\right)$, where $\tau_{w}$ is the wall shear stress (Shields, 1936). It is, therefore, very desirable to include gravitational and buoyant forces in (3) during the contact phase. In our experience, including the gravitational force during contact has a negligible effect in changing the desired $T_{c}$ and $e_{d r y}$. In fact, the results presented so far have all been generated by including buoyant weight during contact.

On the other hand, including the fluid forces during contact can lead to significant drag on the particle throughout the collision. Figure 8 shows how excluding fluid forces during contact gives us excellent agreement with the experimental results, while including fluid forces during contact leads to excessive damping because the fluid surrounding the particle has not been able to adapt to the change of the kinematics of the particle. Indeed, the simulations of Simeonov and Calantoni (2012) show coefficients of restitution below experimental values for moderate Stokes numbers $(20<\mathrm{St}<100)$. Hence, we decided to follow Kempe and Fröhlich (2012a) and to exclude fluid forces during contact for collisions with St $\gg 1$, redefining $(3)$ as follows:

$$
m_{p} \frac{\mathrm{d} \mathbf{u}_{p}}{\mathrm{~d} t}=\left\{\begin{array}{ll}
\mathbf{F}_{h, p}+\mathbf{F}_{g, p}+\mathbf{F}_{c, p} & \zeta_{n}>0 \\
\mathbf{F}_{g, p}+\mathbf{F}_{c, p} & \zeta_{n} \leq 0
\end{array} .\right.
$$


Costa et al. 2015 implemented a similar method for particle-wall collisions, but they turned off fluid forces when the collision overlap exceeded the expected overlap due to the particle's weight, i.e. $\zeta_{n}<-\left(1-\rho_{f} / \rho_{p}\right) g m_{p} / k_{n}$. For the cases shown in Figure 8, the timescale of the contact phase is much smaller

\section{Enduring contact model}

\subsection{Accounting for fluid forces}

As shown in the results from Section 4, neglecting fluid forces acting on the particle during contact produces a good match with the experimental data of Gondret et al. (2002), which involve collisions of finite duration. However, problems can arise in the limit of enduring contact. We therefore propose to include fluid forces during contact below some threshold Stokes number $\mathrm{St}_{\text {crit }}$. For collisions above $\mathrm{St}_{\text {crit }}$, the contact duration should be finite $\left(T_{c}=10 \Delta t\right)$ so 

based on these plots, we selected critical Stokes number $\mathrm{St}_{c r i t}=5$. This value is consistent with the work of other researchers (Gondret et al. 2002, Joseph et al. 2001), who experimentally observed no rebounds for $\mathrm{St}<10$. Note that the Stokes numbers reported in Figure 9 and our resulting St $_{\text {crit }}$ are based on 635 the particle velocity at contact, i.e. when $\zeta_{n}=0$, whereas most other authors

particle is not going to experience an appreciable rebound so that the particle motion is not governed by collision forces during contact, but by hydrodynamic forces. Neglecting hydrodynamic forces in the low-Stokes number regime introduces artifacts in particle mobility. Indeed, this was observed in Vowinckel et al. (2016) for the situation of a horizontal turbulent open-channel flow laden with particles heavier than their critical threshold of motion. Using the same method for collisions, these particles formed a closed bed of resting particles. In this reference, it was shown that a collision with a fast moving particle was necessary for almost all of the erosion events recorded to dislodge a particle out of the sediment packing. However, it has not been possible to clarify to what extent this triggering collision is merely a consequence of the collision procedure.

To investigate what the critical value for the Stokes number may be, we compared particle-wall collisions including hydrodynamic forces during contact ("wet" contact) to those excluding hydrodynamic forces during contact ("dry" contact), as illustrated in Figure 9. For this scenario, we used the same parameters as those summarized in Table $1(\mathrm{St}=27)$ and repeated the simulations for ever-decreasing St. The Stokes number was controlled by prescribing the particle's velocity until it made direct contact with the wall. Unlike the previous simulations, we did not allow the lubrication layer to slow the particle before contact.

For the cases with higher Stokes numbers, we can clearly see how including hydrodynamic forces during contact leads to significant undershooting of the rebound trajectory. As the Stokes number decreases, however, the significance of this undershooting also decreases. For St $<5$ (Figure $9 \mathrm{~d}$ ), there is no appreciable rebound, and we consider the particle to be in enduring contact. Thus, the Stokes numbers reported in Figure 9 and our resulting $\mathrm{St}_{\text {crit }}$ are based on 

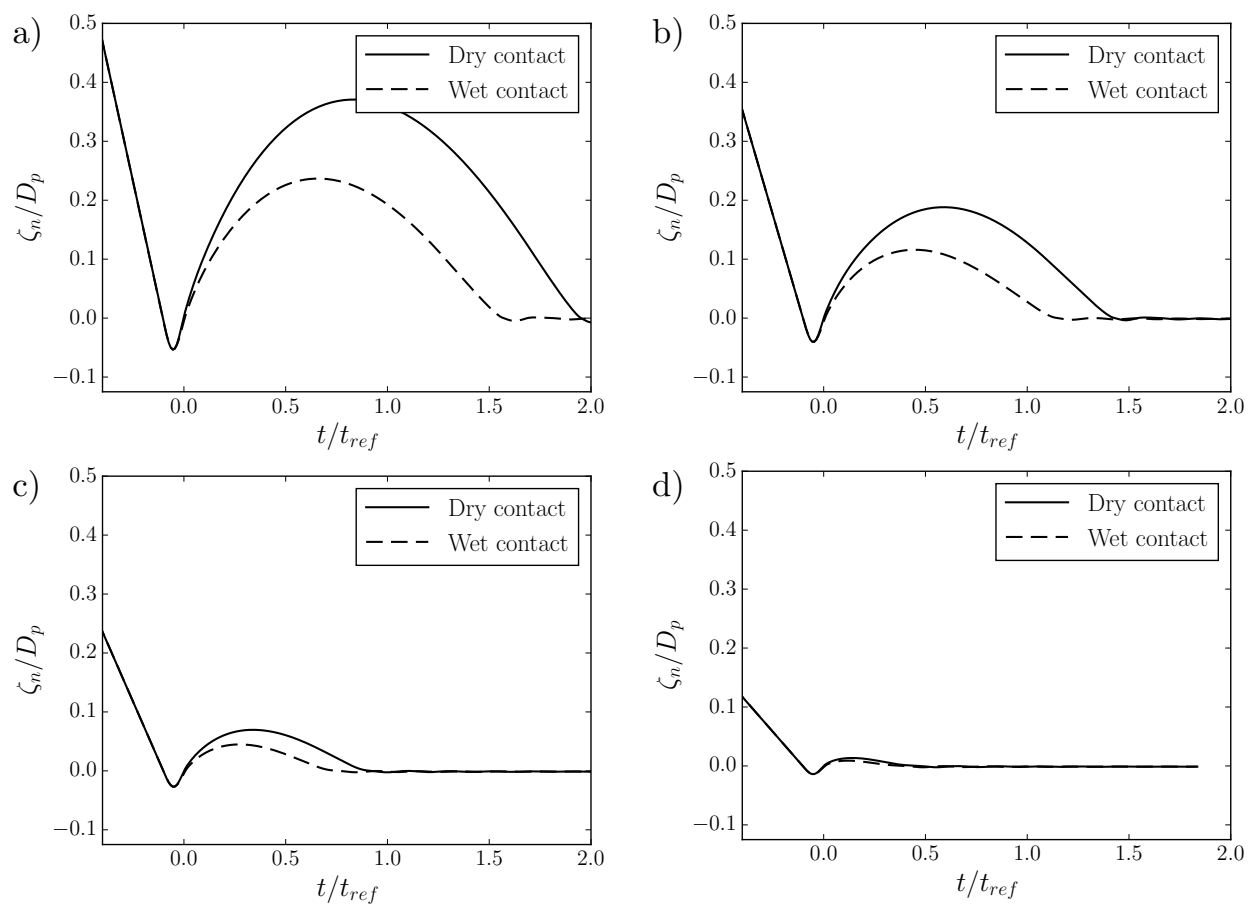

Figure 9: Comparison of trajectories of particle-wall collisions without ('Dry') and with ('Wet') hydrodynamic forces during contact for various Stokes numbers a) St = 20, b) $S t=15$, c) $S t=10, d) S t=5$, where $u_{\text {in }}$ is measured at $\zeta_{n}=0$.

report Stokes numbers at some distance from the wall, before the lubrication layer has fully slowed the particle. With this enduring contact model, we can expand the particle equation of motion 21 to

$$
m_{p} \frac{\mathrm{d} \mathbf{u}_{p}}{\mathrm{~d} t}= \begin{cases}\mathbf{F}_{h, p}+\mathbf{F}_{g, p}+\mathbf{F}_{c, p}, & \zeta_{n}>0 \\ \mathbf{F}_{g, p}+\mathbf{F}_{c, p}, & \zeta_{n} \leq 0 \wedge \max \{\mathrm{St}\}>\mathrm{St}_{c r i t} \\ \mathbf{F}_{h, p}+\mathbf{F}_{g, p}+\mathbf{F}_{c, p}, & \zeta_{n} \leq 0 \wedge \max \{\mathrm{St}\} \leq \mathrm{St}_{c r i t}\end{cases}
$$
among all active collisions for particle $p$, and fluid forces acting on the particle are only included from non-disabled markers. The same consideration applies for the angular momentum (4). Using this scheme now allows us to include the full momentum balance for particles in enduring contact, i.e. the hydrodynamic 645

where the $\max \{\mathrm{St}\}$ function represents the maximum Stokes number collision stresses as well as the buoyant weight of the particle, so that the considerations 
of the Shields parameter become applicable.

\subsection{Optimizing enduring particle overlap}

In the case of $S t \ll 1$, the impact velocity $u_{\text {in }}$ approaches zero. This means in turn that the computed stiffness in (B.1d) would approach infinity. This problem is addressed by Kempe and Fröhlich (2012a) who have introduced a critical Stokes number $\mathrm{St}_{\text {crit }}$, which establishes a minimum impact velocity to limit $k_{n}$ for enduring contact:

$$
u_{i n, c r i t}=\frac{9 \mathrm{St}_{c r i t} \rho_{f} \nu_{f}}{\rho_{p} D_{p}} .
$$

In the present study, this critical impact velocity was used in $(B .1 d)$ and $(B .1 \mathrm{c}$

655 to compute $k_{n}$ and $d_{n}$, respectively, for such collisions. This implementation differs slightly from that of Kempe and Fröhlich (2012a), who do not apply any damping for collisions with $\mathrm{St}<\mathrm{St}_{\text {crit }}$, i.e. they have set $d_{n}=0$. We included this damping for enduring contact in order to help reach steady-state conditions. Our implementation also differs in that we use $\mathrm{St}_{\text {crit }}=5$ whereas Kempe and ${ }_{660}$ Fröhlich (2012a) used St $\mathrm{St}_{\text {crit }}=1$.

Furthermore, we retain the buoyant weight forces in the equation of motion during contact as outlined in Section 4.5. This means that, for particle packings several diameters thick, the weight of a single sphere resting on another layer of particles is passed along to deeper layers. This effect enhances the physical realism because frictional contact forces increase with depth, but it also results in increasing particle surface overlap with depth and ultimately in a change of porosity of the sediment bed, which has been acknowledged as a crucial parameter to define the hydraulic resistance of a sediment to the flow (Vowinckel et al., 2014). However, a flow with a lower Reynolds number would result 670 in collisions with lower Stokes numbers such that $u_{i n, c r i t}$ could become large relative to the particle size and relevant time scales. A large $u_{i n, c r i t}$ would result in a low $k_{n}$ and hence a large overlap between particles, which is undesirable. To prevent this large overlap, we enforce a maximum overlap distance $\epsilon R_{p}$ through the following procedure: for a collision with $\mathrm{St}<\mathrm{St}_{\text {crit }}$, the stiffness is given by 


$$
k_{n}= \begin{cases}\frac{m_{e f f}}{\sqrt{u_{i n} t_{*}^{5}}} & u_{i n}>u_{i n, \text { crit }} \\ \max \left(k_{n, c r i t}, k_{n, \text { grav }}\right) & u_{i n}<u_{i n, \text { crit }}\end{cases}
$$

where $m_{\text {eff }}$ and $t_{*}$ are defined in $\mathrm{A} .2$ and $\mathrm{B} .1 \mathrm{~b}$, respectively,

$$
k_{n, c r i t}=\frac{m_{e f f}}{\sqrt{u_{i n, c r i t} t_{*}^{5}}}
$$

is the stiffness limited by the critical impact velocity, and

$$
k_{n, \text { grav }}=\max \left[m_{p} g\left(\epsilon R_{p}\right)^{-3 / 2}, m_{q} g\left(\epsilon R_{q}\right)^{-3 / 2}\right]
$$

is the stiffness required for particle $p$ (or $q$ ) to have a steady-state overlap of $\epsilon R_{p}$ (or $\epsilon R_{q}$ ) with a wall due to gravity. To have a minimal constant overlap we set $\epsilon=10^{-3}$. Thus, we ensure that a bed of particles contains a uniform set of collision stiffnesses that minimize particle overlap.

\subsection{Rolling and sliding motion}

The coefficient of friction for a material can depend on whether the contact is rolling or sliding (Fishbane et al., 1996). The rolling condition implies zero slip at the contact point, i.e. $\left\|\mathrm{g}_{t, c p}\right\|=0$ (cf. Appendix A). As a consequence, particle surfaces are in sticking contact for rolling motion until a critical threshold of static friction $F_{s}=\mu_{s}\left\|\mathbf{F}_{n}\right\|$ is exceeded, where $\mu_{s}$ is the coefficient of static friction. As soon as this condition is met, significant slip occurs and the contact condition changes from sticking to sliding, so that the threshold for kinetic friction $F_{k}=\mu_{k}\left\|\mathbf{F}_{n}\right\|$ must be used, where $\mu_{k}$ is the coefficient of kinetic friction, with $\mu_{s}$ always greater than $\mu_{k}$. Apart from the physical reasoning presented above, limiting the frictional forces also becomes important from a numerical point of view whenever two or more collision partners are involved. Otherwise the multiple contact points competing for no-slip conditions can lead to instabilities in the calculation of the frictional forces.

In the present study, the distinction between rolling/sticking and sliding is made by the following scheme, which is comparable to that of Luding (2008): 


\begin{tabular}{lcc}
\hline \hline Case & Dry oblique collision & Rolling in shear flow \\
\hline$R_{p}$ & 0.00159 & 0.0625 \\
$\rho_{p} / \rho_{f}$ & 2500 & 2.5 \\
$e_{d r y}$ & 0.83 & 0.97 \\
$\nu$ & 0.22 & 0.3 \\
$\mu_{k}$ & 0.11 & 0.15 \\
$\mu_{s}$ & 0.8 & 0.8 \\
$g$ & 0 & 9.81 \\
$\nu_{f}$ & 0 & 0.02 \\
\hline Timestep & $\Delta t=2 \mathrm{e}-5$ & $\mathrm{CFL}=0.5$ \\
\hline \hline
\end{tabular}

Table 2: Simulation setup for oblique and rolling sphere simulations.

- While the particle is sticking, i.e. $\left\|\mathbf{F}_{t, L S}\right\|<\left\|\mu \mathbf{F}_{n}\right\|$, we set $\mu=\mu_{s}$ to test for the onset of slipping.

- Once slipping occurs, i.e. $\left\|\mathbf{F}_{t, L S}\right\|>\left\|\mu \mathbf{F}_{n}\right\|$, we set $\mu=\mu_{k}$ until the friction force falls below the Coulomb friction force.

705

The aim of the present study is to simulate natural sediment. Hence we parametrized the coefficients of friction with typical values of silicate materials, yielding $\mu_{k}=0.15$ based on the work of Joseph and Hunt (2004), who worked with glass spheres, and $\mu_{s}=0.8$ based on the work of Dieterich (1972), who found values ranging from 0.75 to 0.85 for different rock materials like quartz, granite, and sandstone.

We have validated the tangential collision model using an oblique dry impact experiment, i.e. neglecting hydrodynamic forces, by Foerster et al. (1994), whose parameters are summarized in Table 2, Figure 10 shows that our simulations compare well to the experiments in reproducing the rebound angle

$$
\psi_{\text {out }}=\frac{u_{t, \text { out }}}{u_{n, \text { in }}} \quad \text {, }
$$




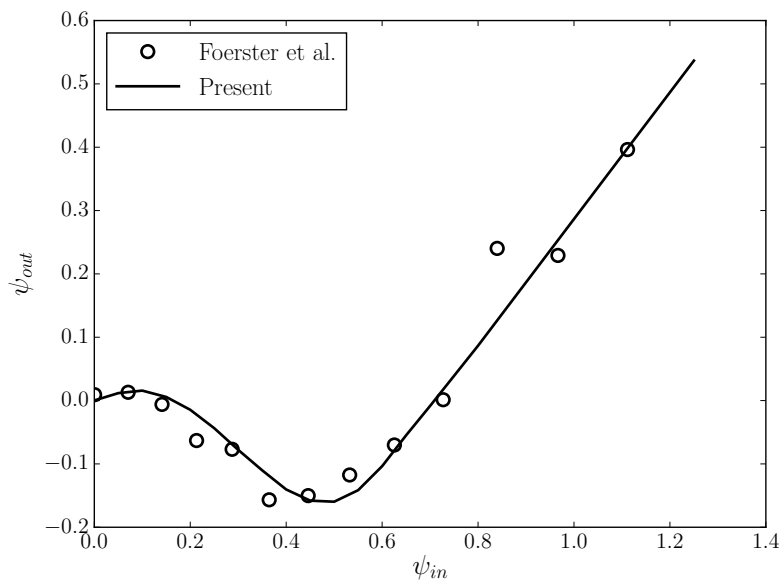

Figure 10: Rebound vs. impact angles for a particle-wall oblique collision.

which depends on the impact angle

$$
\psi_{i n}=\frac{u_{t, i n}}{u_{n, i n}}
$$

Here, $u_{n, \text { in }}$ is the impact velocity normal to the wall, while $u_{t, \text { in }}$ and $u_{t, \text { out }}$ are the impact and rebound velocities, respectively, of the particle's contact point tangential to the wall $\left(u_{t}=u_{p}+R_{p} \omega_{p, z}\right.$ for a particle obliquely colliding in the $x$-direction). For a particle with no initial rotation, $\psi_{i n}$ is the tangent of the angle the particle makes with the wall from the normal $\left(\psi_{i n}=0\right.$ means no relative tangent motion). The rebound angle is zero when the contact is sticking perfectly at the time of release. However, the rebound angle is negative when, at the point of release, $\left|u_{p}\right|<\left|\omega_{p, z}\right|$ (since $\omega_{p, z}<0$ for our example). The linear-spring tangential collision model is able to perfectly capture these negative values for $\psi_{\text {out }}$ at low impact angles.

To test both situations, rolling and sliding, we simulated a particle in a Couette flow. We placed a sphere of radius $R_{p} / H=0.0625$ on the bottom 730 wall of a channel of height $H$. We initialized the particle at rest at a distance $\zeta_{n} / R_{p}=1.6 \times 10^{-5}$ above the bottom wall. We subsequently exposed the sphere to a linear shear flow, holding it fixed for a short time $(t U / H=0.01)$ to allow the flow to develop around it before releasing it. The numerical parameters 

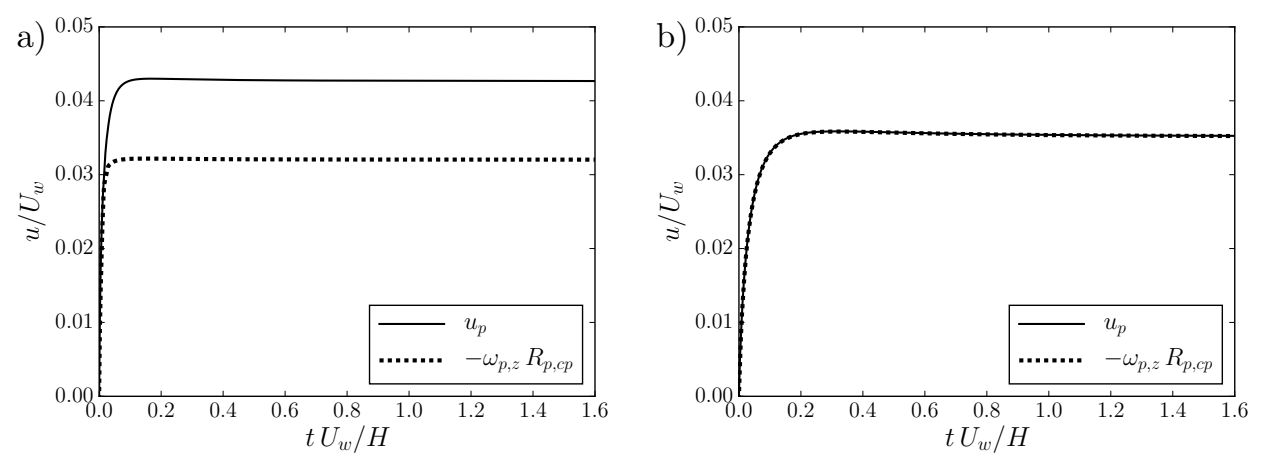

Figure 11: Translational and rotational velocities of a particle exposed to a linear shear flow. a) sliding motion for $R e_{H}=10$ and b) rolling motion for $R e_{H}=50$.

are summarized in Table 2. We found that slipping motion occurs for a lower Reynolds number of $\operatorname{Re}_{H}=U H / \nu_{f}=10$, where $U$ is the lid velocity. On the other hand, perfect rolling motion occurs at $\operatorname{Re}_{H}=U H / \nu_{f}=50$.

Figure 11 shows how the particle accelerates until it reaches a steady-state translational velocity. As soon as the particle makes contact with the wall, gravity holds it there with a slight overlap according to the conditions defined in Section 5.2. Accounting for fluid forces during contact allows the particle to accelerate to a steady-state speed while in contact with the wall. As expected the particle achieves perfect rolling without slip (Figure 11p), marked by the match between the translational velocity $u_{p}$ and the rotational velocity relative to the particle center $-\omega_{p, z} R_{p, c p}$. Accordingly, the particle shows significant slip for the lower Reynolds number (Figure 112), where the increased viscosity leads to increased drag on the particle, which in turn overpowers the friction from the particle's weight.

\section{Flow over dense sediment}

\subsection{Physical setup}

We presented a detailed validation of binary particle-wall collisions in Sections 4 and 5 . To address the bulk behavior of a dense granular bed sheared 

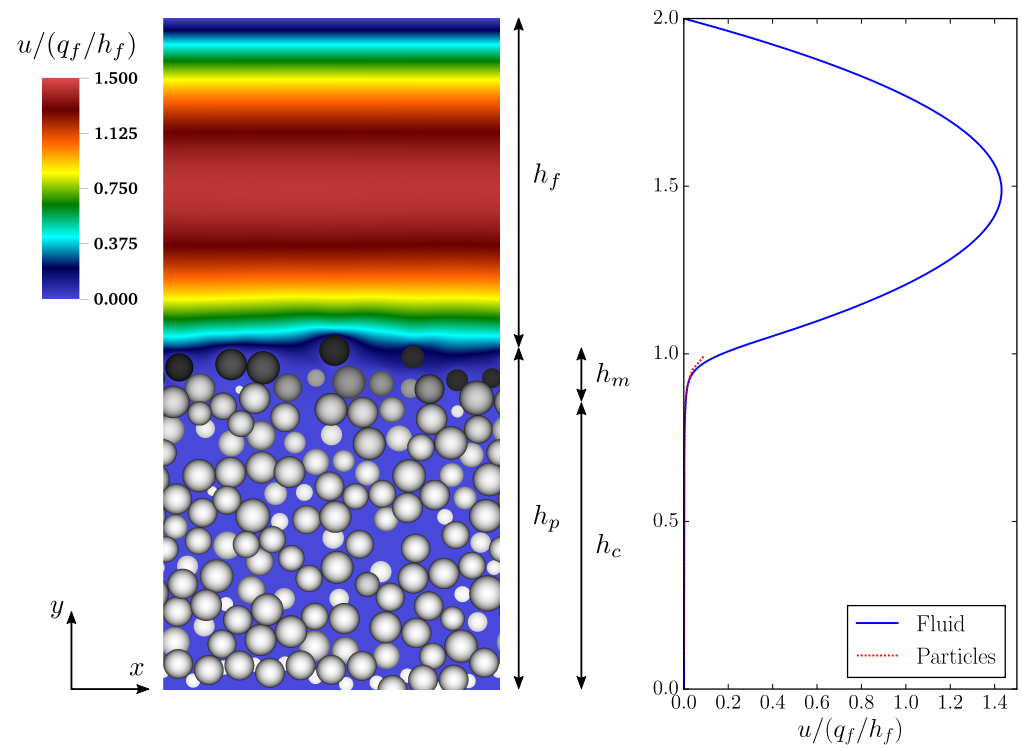

Figure 12: Left plot: Instantaneous snapshot of a slice through the xy-plane for case A10M. Contours show the streamwise component of the fluid velocity. Particles are colored grayscale according to their velocity. Right plot: Streamwise and spanwise averages of fluid and particle velocities. Arrows correspond to the length scales for the clear fluid, $h_{f}$, the particle bed, $h_{p}$, the mobile bed layer, $h_{m}$, and the motionless bed layer, $h_{c}$.

by a laminar Poiseuille flow, we carried out numerical simulations to reproduce the experimental results of Aussillous et al. (2013), who studied pressure-driven flows over glass spheres with a mean diameter $D_{p}=1.1 \mathrm{~mm}$ and and a standard deviation of $\sigma\left(D_{p}\right)=0.1 \mathrm{~mm}$ as sediment material. This experimental work provides investigations over a range of submergences $h_{f} / D_{p}$ and Reynolds numbers in the laminar regime, where $h_{f}$ is the height of the clear-water layer above the sediment bed illustrated in Figure 12. We define $h_{f}$ to be the height above which the average particle volume fraction $\phi<0.05$, which is the threshold for negligible impact of particle-particle interaction on the flow Capart and Fraccarollo, 2011). We define the mobile bed height $h_{m}$ to be the portion of the particle bed above which the mean particle velocity is higher than $1 \%$ of the 


\begin{tabular}{lc}
\hline \hline $\mathrm{Ga}$ & 0.397 \\
\hline$R_{p}(\mathrm{~m})$ & 0.0444 \\
$\rho_{p} / \rho_{f}$ & 2.1 \\
$\nu_{f}\left(\mathrm{~m}^{2} / \mathrm{s}\right)$ & 0.219 \\
$g\left(\mathrm{~m} / \mathrm{s}^{2}\right)$ & 9.81 \\
$e_{d r y}$ & 0.97 \\
$\zeta_{n, \text { min }}(\mathrm{m})$ & $3.0 \times 10^{-3} R_{p}$ \\
$\mu_{k}$ & 0.15 \\
$\mu_{s}$ & 0.8 \\
\hline Domain size $(\mathrm{m})\left(L_{x} \times L_{y} \times L_{z}\right)$ & $1.0 \times 2.0 \times 1.0$ \\
Domain boundary conditions & $\mathrm{p} \times \mathrm{ns} \times \mathrm{p}$ \\
Grid cells in $x$-direction & 256 \\
$D_{p} / h$ & 22.7 \\
Volume fraction in center of bed & 0.609 \\
Timestep & $\mathrm{CFL}=0.1$ \\
\hline \hline
\end{tabular}

Table 3: Simulation parameters to match the experiments of Aussillous et al. (2013). Boundary conditions are periodic (p) and no-slip (ns). The Galileo number Ga is defined in 29 .

value at the fluid/particle interface.

In their experiments, Aussillous et al. (2013) filled a long chamber with particles and then applied a constant pressure gradient, which eroded the particles from the chamber. Initially, the fluid height $h_{f}$ is small and the pressure gradient drives a large number of particles so that the height of the mobile bed layer, $h_{m}$ in Figure 12 is large. Since no new particles are added to the chamber, $h_{f}$ increases as the particles erode away until, at long periods of time, the experiment reaches a steady-state configuration where the influx of particles into the observation window remains in equilibrium with the outflux. Due to our use of periodic boundary conditions, we will only try to replicate the long-term ${ }_{0}$ teady-state flow conditions, of which there are only a few data from Aussillous 


\begin{tabular}{lccccc}
\hline \hline Case & $\mathrm{A} 1$ & $\mathrm{~A} 2$ & $\mathrm{~A} 9$ & $\mathrm{~A} 10$ & $\mathrm{~A} 10 \mathrm{M}$ \\
$\mathrm{Re}_{b}$ & 0.301 & 0.402 & 1.01 & 1.15 & 1.15 \\
$h_{f} / D_{p}$ (Exp.) & $7.05 \pm 0.5$ & $8.15 \pm 0.5$ & $10.29 \pm 0.5$ & $11.27 \pm 0.5$ & $11.27 \pm 0.5$ \\
$h_{f} / D_{p}$ (Sim.) & 7.15 & 8.31 & 10.33 & 11.29 & 11.05 \\
Sh (Exp.) & $0.24 \pm 0.03$ & $0.24 \pm 0.03$ & $0.37 \pm 0.04$ & $0.35 \pm 0.03$ & $0.35 \pm 0.03$ \\
Sh (Sim.) & 0.224 & 0.222 & 0.358 & 0.343 & 0.357 \\
\hline$q_{f}\left(\mathrm{~m}^{2} / \mathrm{s}\right)$ & 0.0659 & 0.0880 & 0.220 & 0.251 & 0.251 \\
$N_{p, m}$ & 2031 & 1870 & 1559 & 1419 & $1407^{*}$ \\
$N_{p, f}$ & 132 & 132 & 132 & 132 & 132 \\
$T_{\text {avg }}(\mathrm{s})$ & 139.5 & 137.9 & 126.2 & 127.3 & 111.0 \\
\hline \hline
\end{tabular}

Table 4: Parameters that vary between the different cases. The bulk Reynolds number $\mathrm{Re}_{b}$ is defined in 30 and the Shields number $\mathrm{Sh}$ is defined in 31. The fluid height $h_{f}$ (and hence Shields number) do not exactly match between the experiments (Exp.) and simulations (Sim.). * Polydisperse particle diameters follow a Gaussian distribution with a standard deviation of $\sigma\left(D_{p}\right)=0.1 D_{p}$.

et al. 2013

775 We executed several simulations in an attempt to match four of the experiments of Aussillous et al. 2013) at different flow rates and fluid heights. The physical and numerical parameters associated with these simulations are listed in Table 3 and the differences between the four cases are listed in Table 4 These experiments can be characterized by the Galileo number

$$
\mathrm{Ga}=\frac{\sqrt{\left(\rho_{p} / \rho_{f}-1\right) g D_{p}^{3}}}{\nu},
$$

the bulk Reynolds number

$$
\operatorname{Re}_{b}=\frac{q_{f}}{\nu} \quad,
$$

where $q_{f}$ is the fluid flow rate, and the Shields number

$$
\mathrm{Sh}=\frac{6 \mathrm{Re}_{b}}{\mathrm{Ga}^{2}}\left(\frac{D_{p}}{h_{f}}\right)^{2},
$$


where $\phi$ is the particle volume fraction. We adopted this procedure because we noticed a hysteresis in the particle flux between an increased flow rate and a decreased flow rate, which has also been observed by Clark et al. 2015). Note that this procedure more closely resembles the experiments, where the particle bed is largely mobilized and then settles into a lower particle flux. 

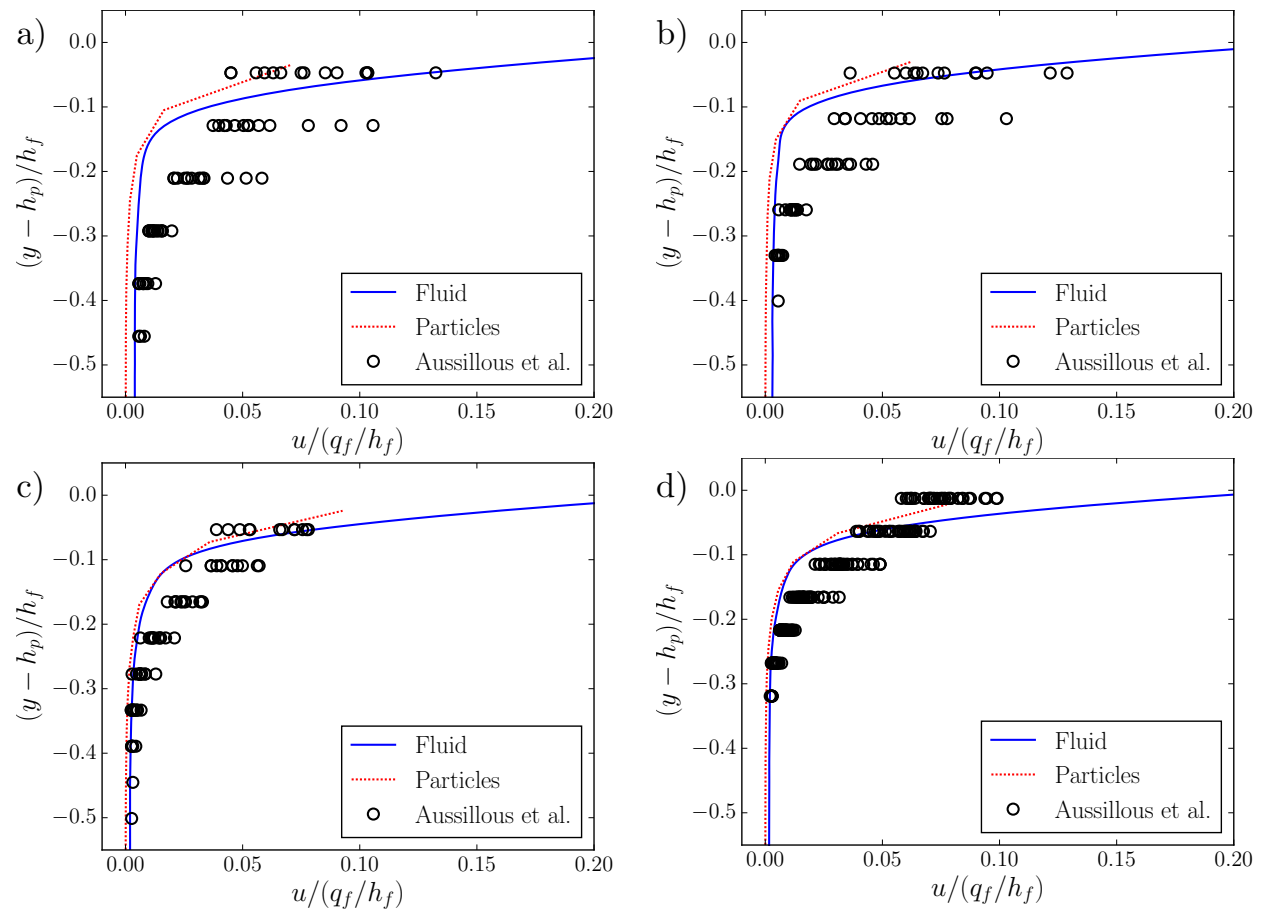

Figure 13: Wall-normal profiles of average fluid and particle velocities near the particle/fluid interface compared to the wall-normal particle velocity profile from Aussillous et al. (2013). a) Case A1, b) Case A2 c) Case A9 d) Case A10.

However, one problem with this procedure is that we cannot determine the final bed height a priori. The dilation and contraction accompanying the two flow rates is difficult to predict without executing an iterative procedure of running simulations with varying numbers of particles. Due to the computational costs of the simulations, we did not iterate on this method, but instead accepted the values we obtained for $h_{f}$, which, with the exception of case A10M, are larger than those in the experiments, as seen in Table 4

\subsection{Comparison of wall-normal profiles}

In Figure 13 we compare the particle velocity profiles of the simulation to the experimental results of Aussillous et al. (2013). We calculated the particle velocity profile $u_{p}(y)$ from our simulations by averaging the velocities of all the particles in the streamwise and spanwise directions whose center fell within a 
given range of heights. We used bins of width $R_{p}$ arranged such that the topmost bin extended from $y=h_{p}-R_{p}$ to $y=h_{p}$. We evaluated the fluid velocity profile by averaging the $u$-velocity field in the streamwise and spanwise directions for each grid cell of the $y$-coordinate. For this calculation, we used the particle cell volume fractions $\phi$ to exclude fluid velocities existing within the particles:

$$
\langle u\rangle_{x z}=\frac{\int_{0}^{L_{z}} \int_{0}^{L_{x}}(1-\phi) u \mathrm{~d} x \mathrm{~d} z}{\int_{0}^{L_{z}} \int_{0}^{L_{x}}(1-\phi) \mathrm{d} x \mathrm{~d} z}
$$

The fluid velocity profiles exhibit a parabolic shape in the clear fluid above the bed, as shown in Figure 12. At the interface between the clear fluid and particle bed, we observe some slip between the fluid and the particles, but within the bed the two velocity profiles are very similar, with only a slight difference due to flow between the particles. The particle velocity profiles from the simulations compare very well with the experiments for cases A9 and A10, and reasonably well for cases A1 and A2.

Part of the discrepancy between our experiments and the simulations is due to the difference in bed height and Shields number as seen in Table 4 . In this table, we can see that cases A1 and A2 exhibit the largest differences in the fluid height between the simulations and experiments, which may have resulted in the larger deviations in the velocity profiles seen in Figure 13. Likewise, for these two cases we can also see larger differences in the Shields number, which can be sensitive to the fluid height $h_{f}$.

\subsection{Comparison of bulk quantities}

We ran the simulation until it reached a constant particle velocity flux $q_{v}$, defined as

$$
q_{v}=\int_{0}^{L_{y}} u_{p}(y) \mathrm{d} y
$$

where $u_{p}(y)$ is the particle velocity profile as defined in the previous section. Unlike $q_{f}$, which had no variability in time, $q_{v}$ did vary as particles occasionally locked in place or rolled over one another. We therefore evaluated a time- 


\begin{tabular}{ccccc}
\hline \hline Case & \multicolumn{3}{c}{ Experimental value } & Simulation value \\
& $\left\langle q_{v}\right\rangle_{T}^{-} / q_{f}$ & $\left\langle q_{v}\right\rangle_{T} / q_{f}$ & $\left\langle q_{v}\right\rangle_{T}^{+} / q_{f}$ & $\left\langle q_{v}\right\rangle_{T} / q_{f}$ \\
\hline $\mathrm{A} 1$ & $5 . \mathrm{e}-3$ & $1.2 \mathrm{e}-2$ & $1.9 \mathrm{e}-2$ & $6.71 \mathrm{e}-3$ \\
\hline $\mathrm{A} 2$ & $5 . \mathrm{e}-3$ & $1.1 \mathrm{e}-2$ & $1.6 \mathrm{e}-2$ & $5.17 \mathrm{e}-3$ \\
\hline $\mathrm{A} 9$ & $4.87 \mathrm{e}-3$ & $8.20 \mathrm{e}-3$ & $1.15 \mathrm{e}-2$ & $7.61 \mathrm{e}-3$ \\
\hline $\mathrm{A} 10$ & $5.17 \mathrm{e}-3$ & $7.04 \mathrm{e}-3$ & $8.91 \mathrm{e}-3$ & $5.84 \mathrm{e}-3$ \\
\hline $\mathrm{A} 10 \mathrm{M}$ & $5.17 \mathrm{e}-3$ & $7.04 \mathrm{e}-3$ & $8.91 \mathrm{e}-3$ & $7.56 \mathrm{e}-3$ \\
\hline \hline
\end{tabular}

Table 5: Comparison of the velocity flux $\left\langle q_{v}\right\rangle_{T}$ between our simulations and the experiments of Aussillous et al. (2013). $\left\langle q_{v}\right\rangle_{T}^{+} / q_{f}$ and $\left\langle q_{v}\right\rangle_{T}^{-} / q_{f}$ represent the mean $\left\langle q_{v}\right\rangle_{T} / q_{f}$ plus and minus the standard deviation over the averaging time, respectively.

averaged value of the particle velocity flux

$$
\left\langle q_{v}\right\rangle_{T}=\frac{1}{T_{a v g}} \int_{t_{s}}^{t_{f}} q_{v} \mathrm{~d} t
$$

where $t_{f}$ is the time at the end of the simulation, $t_{s}$ is the time at which the particle flux reached steady-state, and $T_{a v g}=t_{f}-t_{s}$ is the time interval over which the data was averaged. The values of $T_{\text {avg }}$ are given in Table 4.

In Table 5 , we can see a good agreement between our numerical results and the experimental values of the velocity flux $q_{v}$. Because these quantities are derived from the particle velocity profiles, we expect to see the similar trends, namely that we underestimate the mean values from the experiments and obtain better matches for cases A9 and A10. However, our results still fall within the margin of error of the experiments.

\subsection{Polydisperse flow}

Furthermore, we conducted another simulation to show the effect of multidispersity. In experiments, it is impossible to have a perfectly monodisperse set of particles. In their article, Aussillous et al. (2013) reported having a set of spheres with diameters following a Gaussian distribution of mean $D_{p}=1.1 \mathrm{~mm}$ and standard deviation $\sigma\left(D_{p}\right)=0.1 \mathrm{~mm}$, which is almost $10 \%$ of the mean. We 


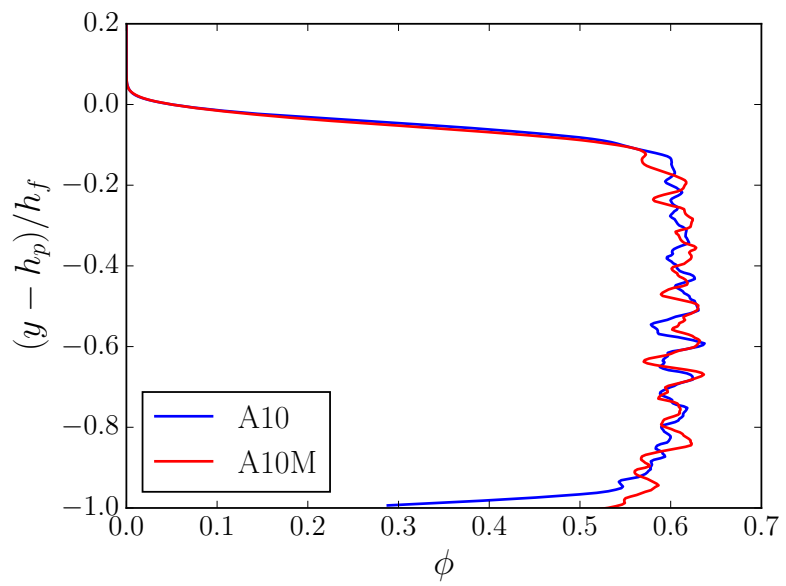

Figure 14: Wall-normal profiles of average particle volume fractions.

created a simulation containing a similar distribution of particle diameters and similar submergence depth to that of case A10. The parameters used are listed under case A10M in Table 4

In Figure 14, we do not see any appreciable changes in the particle bed volume fractions between cases A10 and A10M. The average volume fraction within the bed is $\phi=0.609$, which is consistent with a random sphere packing fraction. On the other hand, in Figure 15 we see a slightly increased velocity profile compared to that of case A10 (Figure 13d). This is likely due to the decreased value of $h_{f}$ compared to that of A10, which results in a higher Shields number, as shown in Table 4. Therefore, we also obtain a velocity flux that overpredicts the mean experimental value, as shown in Table 5 However, the particle velocity profile and velocity flux still agree very well with the experimental results, and the results suggest that using monodisperse spheres is a valid approximation to polydisperse spheres for this experimental setup.

\section{Conclusions}

In the present study, we presented and validated a contact model for the purpose of phase-resolved Direct Numerical Simulations, in which the disperse 


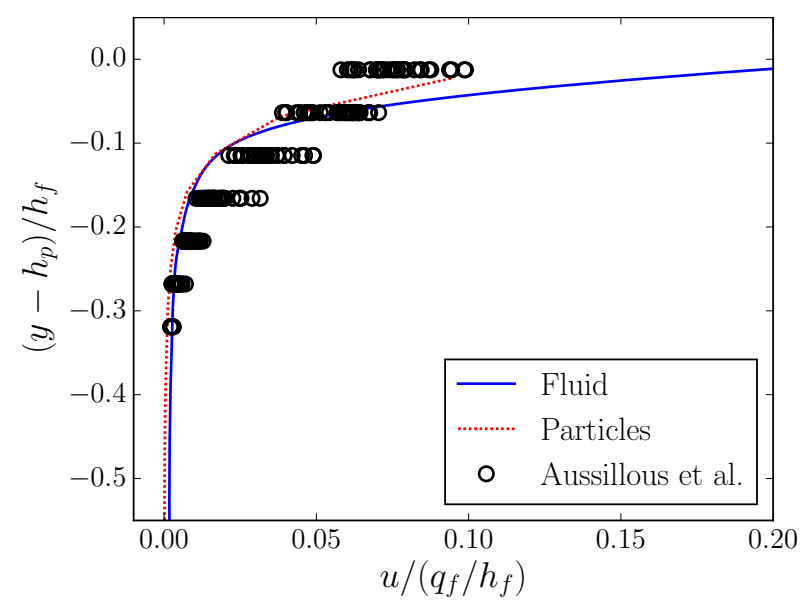

Figure 15: Wall-normal profiles of average fluid and particle velocities for the simulation with polydisperse particles (case A10M).

phase is represented by Immersed Boundary Method. The present modeling approach allows for actual particle contact and takes all relevant contact forces into account without introducing parameters that require arbitrary calibration. These forces include lubrication forces for small inter-particle gaps, normal repulsive forces to resolve inelastic collisions, and tangential forces to represent particle friction. We demonstrated that an improved integration scheme is necessary to obtain consistent results for particle-wall collisions. Subsequently, we presented enhancements that extend the model to deal with simulations of flows over dense granular sediments. It turns out that these enhancements are crucial to deal with thick sediment packings. The measures taken allow us to generate sediment packings several diameters thick that are numerically stable as the packing reaches a steady-state condition. The simulations are performed by retaining the full momentum balance of a particle in enduring contact, which includes the hydrodynamic forces and the buoyant weight of a particle. Including these forces is crucial to represent phenomena like erosion and resuspension of particles. Moreover, the enhanced model allows for rolling and sliding contact, distinguishing between sticking and sliding conditions.

Altogether, the present approach yielded satisfactory agreement with the 
benchmark test cases for binary collisions as well as the collective motion of particles for a horizontal flow over a dense granular packing. In addition, a first test case involving polydisperse sediment was presented. The high degree of accuracy achieved is going to enable us to analyze phase-resolved numerical simulation data in great detail. Although not explicitly stated, we believe that the present approach is also applicable for the situation of vertical channel flows as well as neutrally buoyant particles in laminar and turbulent conditions. It can therefore provide a valuable tool to generate high-fidelity data even on the grain scale of any kind of multiphase flows involving rigid spheres.

\section{Acknowledgements}

This research is supported in part by the Department of Energy Office of Science Graduate Fellowship Program (DOE SCGF), made possible in part by the American Recovery and Reinvestment Act of 2009, administered by ORISEORAU under contract no. DE-AC05-06OR23100. It is also supported by the 20 Petroleum Research Fund, administered by the American Chemical Society, grant number 54948-ND9. BV gratefully acknowledges the Feodor-Lynen scholarship provided by the Alexander von Humboldt foundation, Germany, and EM thanks Petrobras for partial support. The authors thank É. Guazelli and P. Aussillous for stimulating discussions on their experimental work. P. Gondret, N. Mordant, and A. Ten Cate are acknowledged for kindly providing their data. Computational resources for this work used the Extreme Science and Engineering Discovery Environment (XSEDE), which was supported by the National Science Foundation, USA, Grant No. TG-CTS150053.

\section{References}

Apte, S.V., Martin, M., Patankar, N.A., 2009. A numerical method for fully resolved simulation (FRS) of rigid particleflow interactions in complex flows. Journal of Computational Physics 228, 2712-2738. 
Aussillous, P., Chauchat, J., Pailha, M., Médale, M.and Guazzelli, E., 2013. Investigation of the mobile granular layer in bedload transport by laminar shearing flows. Journal of Fluid Mechanics 736, 594-615.

Balachandar, S., Eaton, J.K., 2010. Turbulent dispersed multiphase flow. Annual Review of Fluid Mechanics 42, 111-133.

Breugem, W.P., 2012. A second-order accurate immersed boundary method for fully resolved simulations of particle-laden flows. Journal of Computational Physics 231, 4469-4498.

Capart, H., Fraccarollo, L., 2011. Transport layer structure in intense bed-load. Geophysical Research Letters 38, 2-7.

Charru, F., Andreotti, B., Claudin, P., 2013. Sand ripples and dunes. Annu. Rev. Fluid Mech. 45, 469-493.

${ }_{945}$ Clark, A.H., Shattuck, M.D., Ouellette, N.T., O'Hern, C.S., 2015. Onset and cessation of motion in hydrodynamically sheared granular beds. Physical Review E - Statistical, Nonlinear, and Soft Matter Physics 92, 1-7.

Costa, P., Boersma, B.J., Westerweel, J., Breugem, W.P., 2015. Collision model for fully resolved simulations of flows laden with finite-size particles. Physical Review E 92, 053012.

Cox, R., Brenner, H., 1967. The slow motion of a sphere through a viscous fluid towards a plane surface. Small gap widths, including inertial effects. Chemical Engineering Science 22, 1753-1777.

Derksen, J.J., 2011. Simulations of granular bed erosion due to laminar shear flow near the critical shields number. Physics of Fluids 23, 113303.

Derksen, J.J., 2015. Simulations of granular bed erosion due to a mildly turbulent shear flow. Journal of Hydraulic Research 53, 622-632.

Dieterich, J.H., 1972. Time-dependent friction in rocks. Journal of Geophysical Research 77, 3690-3697. 
Fadlun, E.A., Verzicco, R., Orlandi, P., Mohd-Yusof, J., 2000. Combined immersed-boundary finite-difference methods for three-dimensional complex flow simulations. Journal of Computational Physics 161, 35-60.

Fenton, J.D., Abbott, J.E., 1977. Initial movement of grains on a stream bed - effect of relative protrusion. Proceedings of the Royal Society of London Series A-mathematical Physical and Engineering Sciences 352, 523-537.

Fishbane, P.M., Gasiorowicz, S., Thornton, S.T., 1996. Physics for scientists and engineers. Pearson College Division.

Foerster, S.F., Louge, M.Y., Chang, H., Allia, K., 1994. Measurements of the collision properties of small spheres. Physics of Fluids 6, 1108.

Fukuoka, S., Fukuda, T., Uchida, T., 2014. Effects of sizes and shapes of gravel particles on sediment transports and bed variations in a numerical movablebed channel. Advances in Water Resources 72, 84-96.

Garcia, M., 2008. Sediment transport and morphodynamics, in: Garcia, M. (Ed.), American Society of Civil Engineers, Manuals and Reports on Engineering Practice 110, pp. 21-168.

Glowinski, R., Pan, T.W., Hesla, T.I., Joseph, D.D., 1999. A distributed Lagrange multiplier/fictitious domain method for particulate flows. International Journal of Multiphase Flow 25, 755-794.

Glowinski, R., Pan, T.W., Hesla, T.I., Joseph, D.D., Priaux, J., 2001. A fictitious domain approach to the direct numerical simulation of incompressible viscous flow past moving rigid bodies: Application to particulate flow. Journal Computational Physics 169, 363-426.

Gondret, P., Lance, M., Petit, L., 2002. Bouncing motion of spherical particles in fluids. Physics of Fluids 14, 643-652.

${ }_{985}$ Hertz, H., 1882. Über die Berührung fester elastischer Körper. J. f. reine u. angewandte Math. 92, 156-171. 
Izard, E., Bonometti, T., Lacaze, L., 2014. Modelling the dynamics of a sphere approaching and bouncing on a wall in a viscous fluid. Journal of Fluid Mechanics 747, 422-446.

Joseph, G.G., Hunt, M.L., 2004. Oblique particle-wall collisions in a liquid. Journal of Fluid Mechanics 510, 71-93.

Joseph, G.G., Zenit, R., Hunt, M.L., Rosenwinkel, A.M., 2001. Particle-wall collisions in a viscous fluid. Journal of Fluid Mechanics 433, 329-346.

Kajishima, T., Takiguchi, S., Hamasaki, H., Miyake, Y., 2001. Turbulence structure of particle-laden flow in a vertical plane channel due to vortex shedding. JSME International Journal Series B-Fluids and Thermal Engineering $44,526-535$.

Kempe, T., Fröhlich, J., 2012a. Collision modelling for the interface-resolved simulation of spherical particles in viscous fluids. Journal of Fluid Mechanics $709,445-489$.

Kempe, T., Fröhlich, J., 2012b. An improved immersed boundary method with direct forcing for the simulation of particle laden flows. Journal of Computational Physics 231, 3663-3684.

Kempe, T., Vowinckel, B., Fröhlich, J., 2014. On the relevance of collision modeling for interface-resolving simulations of sediment transport in open channel flow. International Journal of Multiphase Flow 58, 214-235.

Kidanemariam, A.G., Chan-Braun, C., Doychev, T., Uhlmann, M., 2013. Direct numerical simulation of horizontal open channel flow with finite-size, heavy particles at low solid volume fraction. New Journal of Physics 15, 025031.

Kidanemariam, A.G., Uhlmann, M., 2014. Interface-resolved direct numerical simulation of the erosion of a sediment bed sheared by laminar channel flow. International Journal of Multiphase Flow 67, 174-188. 
Lajeunesse, E., Malverti, L., Charru, F., 2010. Bed load transport in turbulent flow at the grain scale: Experiments and modeling. J. Geophys. Res.: Earth Surfarce 115.

Lucci, F., Ferrante, A., Elghobashi, S., 2010. Modulation of isotropic turbulence by particles of taylor length-scale size. Journal of Fluid Mechanics 650, 5-55.

Luding, S., 2008. Cohesive, frictional powders: contact models for tension. Granular Matter 10, 235-246.

Meiburg, E., Kneller, B., 2010. Turbidity currents and their deposits. Annu. Rev. Fluid Mech. 42, 135-156.

Mittal, R., Iaccarino, G., 2005. Immersed boundary methods. Annu. Rev. Fluid Mech. 37, 239-261.

Mordant, N., Pinton, J.F., 2000. Velocity measurement of a settling sphere. The European Physical Journal B-Condensed Matter and Complex Systems 18, 343-352.

Niño, Y., García, M., 1998. Experiments on saltation of sand in water. Journal of Hydraulic Engineering 124, 1014-1025.

Patankar, N.A., Singh, P., Joseph, D.D., Glowinski, R., Pan, T.W., 2000. A new formulation of the distributed Lagrange multiplier/fictitious domain method for particulate flows. International Journal of Multiphase Flow 26, 1509-1524.

Picano, F., Breugem, W.P., Brandt, L., 2015. Turbulent channel flow of dense suspensions of neutrally buoyant spheres. Journal of Fluid Mechanics 764, $463-487$.

Rai, M.M., Moin, P., 1991. Direct simulations of turbulent flow using finitedifference schemes. Journal of computational physics 96, 15-53.

Ray, S., Kempe, T., Fröhlich, 2015. Efficient modelling of particle collisions using a non-linear viscoelastic contact force. International Journal of Multiphase Flow, accepted. 
Roma, A., Peskin, C., Berger, M., 1999. An Adaptive Version of the Immersed Boundary Method. Journal of Computational Physics 153, 509-534.

Santarelli, C., Fröhlich, J., 2015. Direct numerical simulations of spherical bubbles in vertical turbulent channel flow. International Journal of Multiphase Flow 75, 174-193.

Seminara, G., 2010. Fluvial sedimentary patterns. Annu. Rev. Fluid Mech. 42, $43-66$.

Shao, X.M., Wu, T.H., Yu, Z.S., 2012. Fully resolved numerical simulation of particle-laden turbulent flow in a horizontal channel at a low Reynolds number. Journal of Fluid Mechanics 693, 319-344.

Shields, A., 1936. Anwendung der Ähnlichkeitsmechanik und der Turbulenzforschung auf die Geschiebebewegung. Ph.D. thesis. Mitteilungen der Preußischen Versuchsanstalt für Wasserbau und Schiffbau, Berlin (in German).

Sierakowski, A.J., Prosperetti, A., 2016. Resolved-particle simulation by the physalis method: Enhancements and new capabilities. Journal of Computational Physics 309, 164-184.

Simeonov, J.A., Calantoni, J., 2012. Modeling mechanical contact and lubrication in Direct Numerical Simulations of colliding particles. International Journal of Multiphase Flow 46, 38-53.

Ten Cate, A., Nieuwstad, C.H., Derksen, J.J., Van den Akker, H.E.A., 2002. Particle imaging velocimetry experiments and lattice-boltzmann simulations on a single sphere settling under gravity. Physics of Fluids 14, 4012-4025.

Thornton, C., Cummins, S.J., Cleary, P.W., 2011. An investigation of the comparative behaviour of alternative contact force models during elastic collisions. Powder Technology 210, 189-197.

Thornton, C., Cummins, S.J., Cleary, P.W., 2013. An investigation of the comparative behaviour of alternative contact force models during inelastic collisions. Powder Technology 233, 30-46. 
Uhlmann, M., 2005. An immersed boundary method with direct forcing for the simulation of particulate flows. Journal of Computational Physics 209, $448-476$.

Uhlmann, M., 2008. Interface-resolved direct numerical simulation of vertical particulate channel flow in the turbulent regime. Physics of Fluids 20, 053305.

Vowinckel, B., Jain, R., Kempe, T., Fröhlich, J., 2016. Erosion of single particles in a turbulent open-channel flow: a numerical study. J. Hydraul. Res. 54, 158171.

Vowinckel, B., Kempe, T., Fröhlich, J., 2014. Fluid-particle interaction in turbulent open channel flow with fully-resolved mobile beds. Advances in Water Resources 72, 32-44.

Zhang, Z., Prosperetti, A., 2005. A second-order method for three-dimensional 1080 particle simulation. Journal of Computational Physics 210, 292-324.

Zhu, H.P., Zhou, Z.Y., Yang, R.Y., Yu, a.B., 2008. Discrete particle simulation of particulate systems: A review of major applications and findings. Chemical Engineering Science 63, 5728-5770. 


\section{Appendix}

\section{Appendix A. Definitions for particle-particle and particle-wall colli- sions}

In order to discuss collisions in a general manner, we provide definitions for several variables that describe the contact. Some definitions will depend on whether the interaction is between particle $p$ and a wall (particle-wall interaction, or $\mathrm{P}-\mathrm{W}$ ) or between particle $p$ and particle $q$ (particle-particle interaction, or P-P). For most of the definitions, collisions between a fixed particle and a mobile particle are handled identically to particle-particle collisions, unless indicated otherwise (particle-fixed, or P-F).

- $R_{\text {eff }}$ - effective radius

$$
\begin{aligned}
R_{\text {eff }} & =\frac{R_{p} R_{q}}{R_{p}+R_{q}} & & (\mathrm{P}-\mathrm{P}) \\
R_{\text {eff }} & =R_{p} & & (\mathrm{P}-\mathrm{W})
\end{aligned}
$$

- $m_{\text {eff }}$ - effective mass

$$
\begin{aligned}
m_{\text {eff }} & =\frac{m_{p} m_{q}}{m_{p}+m_{q}} & & (\mathrm{P}-\mathrm{P}) \\
m_{\text {eff }} & =m_{p} & & (\mathrm{P}-\mathrm{W}, \mathrm{P}-\mathrm{F})
\end{aligned}
$$

- $\mathbf{x}_{w}$ - point on wall closest to particle.

- $\mathbf{n}$ - unit vector normal to the surface of contact, points from $\mathbf{x}_{p}$ to $\mathbf{x}_{q}$ (P-P) or directly towards the wall (P-W).

$$
\begin{aligned}
& \mathbf{n}=\frac{\mathbf{x}_{q}-\mathbf{x}_{p}}{\left\|\mathbf{x}_{q}-\mathbf{x}_{p}\right\|} \quad(\mathrm{P}-\mathrm{P}) \\
& \mathbf{n}=\frac{\mathbf{x}_{w}-\mathbf{x}_{p}}{\left\|\mathbf{x}_{w}-\mathbf{x}_{p}\right\|} \quad(\mathrm{P}-\mathrm{W})
\end{aligned}
$$

- $\zeta_{n}$ - distance between surfaces of the two bodies (negative value indicates overlap).

$$
\begin{aligned}
& \zeta_{n}=\left\|\mathbf{x}_{q}-\mathbf{x}_{p}\right\|-R_{p}-R_{q} \quad(\mathrm{P}-\mathrm{P}) \\
& \zeta_{n}=\left\|\mathbf{x}_{w}-\mathbf{x}_{p}\right\|-R_{p} \quad(\mathrm{P}-\mathrm{W})
\end{aligned}
$$


- $\mathbf{x}_{c p}$ - location of contact point between surfaces, halfway between surface overlap (P-P).

$$
\begin{aligned}
& \mathbf{x}_{c p}=\mathbf{x}_{p}+\left(R_{p}+\frac{\zeta_{n}}{2}\right) \mathbf{n} \\
& \mathbf{x}_{c p}=\mathbf{x}_{w}
\end{aligned}
$$

- $R_{p, c p}$ - radius of particle $p$ with respect to the contact point.

$$
R_{p, c p}=\left\|\mathbf{x}_{c p}-\mathbf{x}_{p}\right\|
$$

- $\mathbf{g}$ - relative velocity between particle centers of mass.

$$
\begin{array}{ll}
\mathbf{g}=\mathbf{u}_{p}-\mathbf{u}_{q} & (\mathrm{P}-\mathrm{P}) \\
\mathbf{g}=\mathbf{u}_{p} & (\mathrm{P}-\mathrm{W})
\end{array}
$$

- $\mathbf{g}_{c p}$ - relative velocity of surface contact point.

$$
\begin{array}{ll}
\mathbf{g}_{c p}=\mathbf{g}+R_{p, c p}\left(\boldsymbol{\omega}_{p} \times \mathbf{n}\right)+R_{q, c p}\left(\boldsymbol{\omega}_{q} \times \mathbf{n}\right) & (\mathrm{P}-\mathrm{P}) \\
\mathbf{g}_{c p}=\mathbf{g}+R_{p, c p}\left(\boldsymbol{\omega}_{p} \times \mathbf{n}\right) & (\mathrm{P}-\mathrm{W})
\end{array}
$$

- $\mathbf{g}_{n, c p}$ - component of $\mathbf{g}_{c p}$ normal to surface.

$$
\mathbf{g}_{n, c p}=\left(\mathbf{g}_{c p} \cdot \mathbf{n}\right) \mathbf{n}
$$

- $\mathbf{g}_{t, c p}$ - component of $\mathbf{g}_{c p}$ tangent to surface.

$$
\mathbf{g}_{t, c p}=\mathbf{g}_{c p}-\mathbf{g}_{n, c p}
$$

\section{Appendix B. Calculating the normal contact model coefficients}

In order to obtain the stiffness and damping coefficients $k_{n}$ and $d_{n}$, Ray et al. (2015) use nonlinear transformations and a series expansion of 111 to yield the following algebraic expressions:

$$
\lambda=\frac{1}{\alpha^{2} \tau_{c, 0}^{2}}\left(-\frac{1}{2} C \eta+\sqrt{\frac{1}{4} C^{2} \eta^{2}+\alpha^{2} \tau_{c, 0}^{2} \eta}\right),
$$




$$
\begin{gathered}
t_{*}=\frac{T_{c}}{\tau_{c, 0}} \sqrt{1-A \lambda-B \lambda^{2}}, \\
d_{n}=\frac{2 \lambda m_{\text {eff }}}{t_{*}},
\end{gathered}
$$

1105 and

$$
k_{n}=\frac{m_{\text {eff }}}{\sqrt{u_{i n} t_{*}^{5}}},
$$

where $A=0.716, B=0.830, C=0.744, \alpha=1.111$, and $\tau_{c, 0}=3.218$ are constants. The parameter $\eta=\left(\ln e_{d r y}\right)^{2}$ accounts for the restitution coefficient, and we measure the impact velocity to be $u_{i n}=\mathbf{g}_{n, c p} \cdot \mathbf{n}$ at the first occurrence of $\zeta_{n} \leq 0$.

\section{Appendix C. The tangential displacement vector}

Tangential models based on spring systems require a displacement as defined by (13), which represents the accumulated relative motion between two surfaces We calculate $\boldsymbol{\zeta}_{t}$ in a discrete sense as follows:

$$
\begin{aligned}
\widetilde{\boldsymbol{\zeta}}_{t} & =\boldsymbol{\zeta}_{t}^{k-1}-\left(\boldsymbol{\zeta}_{t}^{k-1} \cdot \mathbf{n}\right) \mathbf{n} \\
\widehat{\boldsymbol{\zeta}}_{t} & =\frac{\left\|\boldsymbol{\zeta}_{t}^{k-1}\right\| \widetilde{\boldsymbol{\zeta}}_{t}}{\left\|\widetilde{\boldsymbol{\zeta}}_{t}\right\|} \\
\boldsymbol{\zeta}_{t}^{k} & =\widehat{\boldsymbol{\zeta}}_{t}+2 \alpha_{k} \Delta t \mathbf{g}_{t, c p}
\end{aligned}
$$

Equations (C.1a) and (C.1b) rotate the displacement from the previous timestep onto a plane tangent to the two surfaces. Luding (2008) implemented this rotation to account for the change in reference frame that can take place between two timesteps. Without this rotation, the linear spring could contribute to the normal force acting between two particles.

Furthermore, when the two surfaces slip according to the Coulomb friction criteria, the displacement vector should not grow as the two surfaces continue to slide past one another. Instead, we reset the displacement to that which achieves the Coulomb friction force:

$$
\boldsymbol{\zeta}_{t}=-\frac{\left\|\mu \mathbf{F}_{n}\right\| \mathbf{t}+d_{t} \mathbf{g}_{t, c p}}{k_{t}} \quad \text { if } \quad\left\|\mathbf{F}_{t, L S}\right\|>\left\|\mu \mathbf{F}_{n}\right\|
$$

